\title{
Universal Bethe Ansatz and Scalar Products of Bethe Vectors ${ }^{\star}$
}

\author{
Samuel BELLIARD ${ }^{\dagger}$, Stanislav PAKULIAK ${ }^{\ddagger}$ and Eric RAGOUCY $Y^{\S}$ \\ † Istituto Nazionale di Fisica Nucleare, Sezione di Bologna, Italy \\ E-mail: belliard@bo.infn.it \\ ¥ Institute of Theoretical \& Experimental Physics, 117259 Moscow, Russia \\ Laboratory of Theoretical Physics, JINR, 141980 Dubna, Moscow reg., Russia \\ Moscow Institute of Physics and Technology, 141700, Dolgoprudny, Moscow reg., Russia \\ E-mail: pakuliak@theor.jinr.ru \\ $\S$ Laboratoire de Physique Théorique LAPTH, CNRS and Université de Savoie, \\ BP 110, 74941 Annecy-le-Vieux Cedex, France \\ E-mail: eric.ragoucy@lapp.in2p3.fr
}

Received October 25, 2010; Published online December 14, 2010

doi:10.3842/SIGMA.2010.094

\begin{abstract}
An integral presentation for the scalar products of nested Bethe vectors for the quantum integrable models associated with the quantum affine algebra $U_{q}\left(\widehat{\mathfrak{g l}}_{3}\right)$ is given. This result is obtained in the framework of the universal Bethe ansatz, using presentation of the universal Bethe vectors in terms of the total currents of a "new" realization of the quantum affine algebra $U_{q}\left(\widehat{\mathfrak{g l}}_{3}\right)$.
\end{abstract}

Key words: Bethe ansatz; quantum affine algebras

2010 Mathematics Subject Classification: 17B37; 81R50

\section{Introduction}

The problem of computing correlation functions is one of the most challenging problem in the field of quantum integrable models, starting from the establishment of the Bethe ansatz method in [1]. For the models where algebraic Bethe ansatz [2, 3, 4, 5] is applicable, this problem can be reduced to the calculation of scalar products of off-shell Bethe vectors. These latters are Bethe vectors where the Bethe parameters are not constrained to obey the Bethe Ansatz equations anymore. For $\mathfrak{g l}_{2}$-based integrable models, these scalar products were calculated in $[6,7,8]$ and are given by the sums over partitions of two sets of the Bethe parameters. Lately, it was shown by N. Slavnov [9], that if one set of Bethe parameters satisfies Bethe equations (which guarantees that the Bethe vectors are eigenvectors of the transfer matrix), then the formula for scalar products can be written in a determinant form. This form is very useful to get an integral presentation for correlation functions [10, 11, 12, 13] in the thermodynamic limit.

There is a wide class of quantum integrable models associated with the algebra $\mathfrak{g l}_{N}(N>2)$. An algebraic Bethe ansatz for these type models is called hierarchical (or nested) and was introduced by P. Kulish and N. Reshetikhin [14]. This method is based on a recursive procedure which reduces the eigenvalue problem for the transfer matrix for the model with $\mathfrak{g l}_{N}$ symmetry to an analogous problem for the model with $\mathfrak{g l}_{N-1}$ symmetry. Assuming that the problem for

\footnotetext{
${ }^{\star}$ This paper is a contribution to the Proceedings of the International Workshop "Recent Advances in Quantum Integrable Systems". The full collection is available at http://www.emis.de/journals/SIGMA/RAQIS2010.html
} 
$N=2$ is solved, this method allows to find hierarchical Bethe equations. Explicit formulas for the hierarchical Bethe vectors in terms of the matrix elements of the $\mathfrak{g l}_{N}$ monodromy matrix can be found in [15], but these complicated expressions are very difficult to handle.

The solution of this problem, namely the formulas for the off-shell Bethe vectors in terms of monodromy matrix, was found in [16]. These vectors are called universal, because they have the same structure for the different models sharing the same hidden symmetry. This construction requires a very complicated procedure of calculation of the trace of projected tensor powers of the monodromy matrix. It was performed in [17], but only on the level of the evaluation representation of $U_{q}\left(\widehat{\mathfrak{g l}}_{N}\right)$ monodromy matrix.

There is a new, alternative approach to the construction of universal Bethe vectors for $\mathfrak{g l}_{N}$ symmetry models using current realizations of the quantum affine algebras [18] and using a Ding-Frenkel isomorphism between current and L-operators realizations of the quantum affine algebra $U_{q}\left(\widehat{\mathfrak{g l}}_{N}\right)$ [19]. This approach allows to obtain explicit formulas for the universal Bethe vectors in terms of the current generators of the quantum affine algebra $U_{q}\left(\widehat{\mathfrak{g l}}_{N}\right)$ for arbitrary highest weight representations. It was proved in [20] that the two methods of construction of the universal Bethe vectors coincide on the level of the evaluation representations. Furthermore, it was shown in [21] that the eigenvalue property of the hierarchical universal Bethe vectors can be reformulated as a problem of ordering of the current generators in the product of the universal transfer matrix and the universal Bethe vectors. It was proved that the eigenvalue property appears only if parameters of the universal Bethe vectors satisfy the universal Bethe equations of the analytical Bethe ansatz [22].

The universal Bethe vectors in terms of current generators have an integral presentation, as

an integral transform with some kernel of the product of the currents. In the $U_{q}\left(\widehat{\mathfrak{s l}}_{2}\right)$ case, this integral representation produces immediately an integral formula for the scalar product of offshell Bethe vectors [23] which is equivalent to the Izergin-Korepin formula. In this article, we present an integral presentation of the universal off-shell Bethe vectors based on the quantum affine algebra $U_{q}\left(\widehat{\mathfrak{g l}}_{3}\right)$. These integral formulas lead to integral formulas for scalar products with some kernel. The corresponding formula (5.6) is the main result of our paper. The problem left to be done is to transform the integral form we have obtained to a determinant form which can be very useful for the application to quantum integrable models associated to $\mathfrak{g l}_{N}$ symmetry algebra.

\section{Universal Bethe vectors in terms of L-operator}

\section{$2.1 U_{q}\left(\widehat{\mathfrak{g l}}_{3}\right)$ in L-operator formalism}

Let $\mathrm{E}_{i j} \in \operatorname{End}\left(\mathbb{C}^{3}\right)$ be a matrix with the only nonzero entry equals to 1 at the intersection of the $i$-th row and $j$-th column. Let $\mathrm{R}(u, v) \in \operatorname{End}\left(\mathbb{C}^{3} \otimes \mathbb{C}^{3}\right) \otimes \mathbb{C}[[v / u]]$,

$$
\begin{aligned}
\mathrm{R}(u, v)= & \sum_{1 \leq i \leq 3} \mathrm{E}_{i i} \otimes \mathrm{E}_{i i}+\frac{u-v}{q u-q^{-1} v} \sum_{1 \leq i<j \leq 3}\left(\mathrm{E}_{i i} \otimes \mathrm{E}_{j j}+\mathrm{E}_{j j} \otimes \mathrm{E}_{i i}\right) \\
& +\frac{q-q^{-1}}{q u-q^{-1} v} \sum_{1 \leq i<j \leq 3}\left(u \mathrm{E}_{i j} \otimes \mathrm{E}_{j i}+v \mathrm{E}_{j i} \otimes \mathrm{E}_{i j}\right)
\end{aligned}
$$

be a trigonometric R-matrix associated with the vector representation of $\mathfrak{g l}_{3}$. Let $q$ be a complex parameter different from zero or a root of unity.

The algebra $U_{q}\left(\widehat{\mathfrak{g l}}_{3}\right)$ (with zero central charge and the gradation operator dropped out) is an associative algebra with unit, generated by the modes $\mathrm{L}_{i, j}^{ \pm}[ \pm k], k \geq 0,1 \leq i, j \leq 3$ of the 
L-operators $\mathrm{L}^{ \pm}(z)=\sum_{k=0}^{\infty} \sum_{i, j=1}^{3} \mathrm{E}_{i j} \otimes \mathrm{L}_{i, j}^{ \pm}[ \pm k] z^{\mp k}$, subject to the relations

$$
\begin{aligned}
& \mathrm{R}(u, v) \cdot\left(\mathrm{L}^{ \pm}(u) \otimes \mathbf{1}\right) \cdot\left(\mathbf{1} \otimes \mathrm{L}^{ \pm}(v)\right)=\left(\mathbf{1} \otimes \mathrm{L}^{ \pm}(v)\right) \cdot\left(\mathrm{L}^{ \pm}(u) \otimes \mathbf{1}\right) \cdot \mathrm{R}(u, v), \\
& \mathrm{R}(u, v) \cdot\left(\mathrm{L}^{+}(u) \otimes \mathbf{1}\right) \cdot\left(\mathbf{1} \otimes \mathrm{L}^{-}(v)\right)=\left(\mathbf{1} \otimes \mathrm{L}^{-}(v)\right) \cdot\left(\mathrm{L}^{+}(u) \otimes \mathbf{1}\right) \cdot \mathrm{R}(u, v), \\
& \mathrm{L}_{j, i}^{+}[0]=\mathrm{L}_{i, j}^{-}[0]=0, \quad 1 \leq i<j \leq 3, \\
& \mathrm{~L}_{k, k}^{+}[0] \mathrm{L}_{k, k}^{-}[0]=1, \quad 1 \leq k \leq 3 .
\end{aligned}
$$

Actually, we will not impose the condition (2.3) since the universal Bethe vectors will be constructed only from one L-operator, say $\mathrm{L}^{+}(z)$.

Subalgebras formed by the modes $\mathrm{L}^{ \pm}[n]$ of the L-operators $\mathrm{L}^{ \pm}(z)$ are the standard Borel subalgebras $U_{q}\left(\mathfrak{b}^{ \pm}\right) \subset U_{q}\left(\widehat{\mathfrak{g l}}_{N}\right)$. These Borel subalgebras are Hopf subalgebras of $U_{q}\left(\widehat{\mathfrak{g l}}_{N}\right)$. Their coalgebraic structure is given by the formulae

$$
\Delta\left(\mathrm{L}_{i, j}^{ \pm}(u)\right)=\sum_{k=1}^{N} \mathrm{~L}_{k, j}^{ \pm}(u) \otimes \mathrm{L}_{i, k}^{ \pm}(u) .
$$

\subsection{Universal off-shell Bethe vectors}

We will follow the construction of the off-shell Bethe vectors due to [16]. Let L $(z)=\sum_{k=0}^{\infty} \sum_{i, j=1}^{3} \mathrm{E}_{i j} \otimes$ $\mathrm{L}_{i, j}[k] z^{-k}$ be the L-operator ${ }^{1}$ of the Borel subalgebra $U_{q}\left(\mathfrak{b}^{+}\right)$of $U_{q}\left(\widehat{\mathfrak{g l}}_{3}\right)$ satisfying the YangBaxter commutation relation with a $R$-matrix $\mathrm{R}(u, v)$. We use the notation $\mathrm{L}^{(k)}(z) \in\left(\mathbb{C}^{3}\right)^{\otimes M} \otimes$ $U_{q}\left(\mathfrak{b}^{+}\right)$for L-operator acting nontrivially on $k$-th tensor factor in the product $\left(\mathbb{C}^{3}\right)^{\otimes M}$ for $1 \leq k \leq M$. Consider a series in $M$ variables

$$
\mathbb{T}\left(u_{1}, \ldots, u_{M}\right)=\mathrm{L}^{(1)}\left(u_{1}\right) \cdots \mathrm{L}^{(M)}\left(u_{M}\right) \cdot \mathbb{R}^{(M, \ldots, 1)}\left(u_{M}, \ldots, u_{1}\right),
$$

with coefficients in $\left(\operatorname{End}\left(\mathbb{C}^{3}\right)\right)^{\otimes M} \otimes U_{q}\left(\mathfrak{b}^{+}\right)$, where

$$
\mathbb{R}^{(M, \ldots, 1)}\left(u_{M}, \ldots, u_{1}\right)=\prod_{M \geq j>1} \prod_{j>i \geq 1} \mathrm{R}^{(j i)}\left(u_{j}, u_{i}\right)
$$

In the ordered product of $R$-matrices $(2.5)$, the $\mathrm{R}^{(j i)}$ factor is on the left of the $\mathrm{R}^{(\mathrm{ml})}$ factor if $j>m$, or $j=m$ and $i>l$. Consider the set of variables

$$
\{\bar{t}, \bar{s}\}=\left\{t_{1}, \ldots, t_{a} ; s_{1}, \ldots, s_{b}\right\}
$$

Following [16], let

$$
\begin{aligned}
\mathbb{B}(\bar{t}, \bar{s})= & \prod_{1 \leq j \leq b} \prod_{1 \leq i \leq a} \frac{q s_{j}-q^{-1} t_{i}}{s_{j}-t_{i}} \\
& \times\left(\operatorname{tr}_{\left(\mathbb{C}^{3}\right) \otimes(a+b)} \otimes \mathrm{id}\right)\left(\mathbb{T}\left(t_{1}, \ldots, t_{a} ; s_{1}, \ldots, s_{b}\right) \mathrm{E}_{21}^{\otimes a} \otimes \mathrm{E}_{32}^{\otimes b} \otimes 1\right) .
\end{aligned}
$$

The element $\mathbb{T}(\bar{t}, \bar{s})$ in (2.6) is given by (2.4) with obvious identification. The coefficients of $\mathbb{B}(\bar{t}, \bar{s})$ are elements of the Borel subalgebra $U_{q}\left(\mathfrak{b}^{+}\right)$.

\footnotetext{
${ }^{1}$ We omit superscript + in this L-operator, since will consider only positive standard Borel subalgebra $U_{q}\left(\mathfrak{b}^{+}\right)$ here and below.
} 
We call vector $v$ a right weight singular vector if it is annihilated by any positive mode $\mathrm{L}_{i, j}[n]$, $i>j, n \geq 0$ of the matrix elements of the $\mathrm{L}^{+}(z)$ operator and is an eigenvector of the diagonal matrix entries $\mathrm{L}_{i, i}^{+}(z)$ :

$$
\mathrm{L}_{i, j}^{+}(z) v=0, \quad i>j, \quad \mathrm{~L}_{i, i}^{+}(z) v=\lambda_{i}(z) v, \quad i=1, \ldots, 3 .
$$

For any right $U_{q}\left(\widehat{\mathfrak{g l}}_{3}\right)$-module $V$ with a right singular vector $v$, denote

$$
\mathbb{B}_{V}(\bar{t}, \bar{s})=\mathbb{B}(\bar{t}, \bar{s}) v \text {. }
$$

The vector valued function $\mathbb{B}_{V}(\bar{t}, \bar{s})$ was called in $[16,17]$ universal off-shell Bethe vector.

We call vector $v^{\prime}$ a left weight singular vector if it is annihilated by any positive mode $\mathrm{L}_{i, j}[n]$, $i<j, n \geq 0$ of the matrix elements of the L-operator $\mathrm{L}^{+}(z)$ and is an eigenvector of the diagonal matrix entries $\mathrm{L}_{i, i}^{+}(z)$ :

$$
0=v^{\prime} \mathrm{L}_{i, j}^{+}(z), \quad i<j, \quad v^{\prime} \mathrm{L}_{i, i}^{+}(z)=\mu_{i}(z) v^{\prime}, \quad i=1, \ldots, 3 .
$$

For any left $U_{q}\left(\widehat{\mathfrak{g l}}_{3}\right)$-module $V^{\prime}$ with a left singular vector $v^{\prime}$, denote

$$
\mathbb{C}_{V^{\prime}}(\bar{\tau}, \bar{\sigma})=v^{\prime} \mathbb{C}(\bar{\tau}, \bar{\sigma})
$$

where

$$
\{\bar{\tau}, \bar{\sigma}\}=\left\{\tau_{1}, \ldots, \tau_{a} ; \sigma_{1}, \ldots, \sigma_{b}\right\},
$$

and

$$
\begin{aligned}
\mathbb{C}(\bar{\tau}, \bar{\sigma})= & \prod_{1 \leq j \leq b} \prod_{1 \leq i \leq a} \frac{q \sigma_{j}-q^{-1} \tau_{i}}{\sigma_{j}-\tau_{i}} \\
& \times\left(\operatorname{tr}_{\left(\mathbb{C}^{3}\right) \otimes(a+b)} \otimes \mathrm{id}\right)\left(\mathbb{T}\left(\tau_{1}, \ldots, \tau_{a} ; \sigma_{1}, \ldots, \sigma_{b}\right) \mathrm{E}_{12}^{\otimes a} \otimes \mathrm{E}_{23}^{\otimes b} \otimes 1\right) .
\end{aligned}
$$

Our goal is to calculate the scalar product

$$
\left\langle\mathbb{C}_{V^{\prime}}(\bar{\tau}, \bar{\sigma}), \mathbb{B}_{V}(\bar{t}, \bar{s})\right\rangle
$$

There is a direct way to solve this problem, using the exchange relations of the L-operators matrix elements and the definitions of the singular weight vectors. However, this approach is a highly complicated combinatorial problem. Instead, we will use another presentation of the universal Bethe vectors given recently in the paper [20, 24], using current realization of the quantum affine algebra $U_{q}\left(\widehat{\mathfrak{g l}}_{3}\right)$ and method of projections introduced in [25] and developed in $[26]$.

\section{Current realization of $U_{q}\left(\widehat{\mathfrak{g l}}_{3}\right)$}

\subsection{Gauss decompositions of L-operators}

The relation between the L-operator realization of $U_{q}\left(\widehat{\mathfrak{g l}}_{3}\right)$ and its current realization [18] is known since the work [19]. To build an isomorphism between these two realizations, one has to consider the Gauss decomposition of the L-operators and identifies linear combinations of some Gauss coordinates with the total currents of $U_{q}\left(\widehat{\mathfrak{g l}}_{3}\right)$ corresponding to the simple roots of $\mathfrak{g l}_{3}$. Recently, it was shown in [24] that there are two different but isomorphic current realization of $U_{q}\left(\widehat{\mathfrak{g l}}_{3}\right)$. They correspond to different embeddings of smaller algebras into bigger ones and to different type of Gauss decompositions of the fundamental L-operators. These two different 
current realizations have different commutation relations, different current comultiplications and different associated projections onto intersections of the current and Borel subalgebras of $U_{q}\left(\widehat{\mathfrak{g l}}_{3}\right)$.

Our way to calculate the scalar product of Bethe vectors (2.11) is to use an alternative form to expressions (2.8) and (2.10) for the universal Bethe vectors. It is written in terms of projections of products of currents onto intersections of the current and Borel subalgebras of $U_{q}\left(\widehat{\mathfrak{g l}}_{3}\right)$. In this case, the universal Bethe vectors can be written as some integral and the calculation of the scalar product is reduced to the calculation of an integral of some rational function.

For the L-operators fixed by the relations (2.1) and (2.2), we consider the following decompositions into Gauss coordinates $\mathrm{F}_{j, i}^{ \pm}(t), \mathrm{E}_{i, j}^{ \pm}(t), j>i$ and $k_{i}^{ \pm}(t)$ :

$$
\begin{aligned}
& \mathrm{L}_{i, j}^{ \pm}(t)=\mathrm{F}_{j, i}^{ \pm}(t) k_{i}^{+}(t)+\sum_{1 \leq m<i} \mathrm{~F}_{j, m}^{ \pm}(t) k_{m}^{ \pm}(t) \mathrm{E}_{m, i}^{ \pm}(t), \quad 1 \leq i<j \leq 3, \\
& \mathrm{~L}_{i, i}^{ \pm}(t)=k_{i}^{ \pm}(t)+\sum_{1 \leq m<i} \mathrm{~F}_{i, m}^{ \pm}(t) k_{m}^{ \pm}(t) \mathrm{E}_{m, i}^{ \pm}(t), \quad i=1,2,3, \\
& \mathrm{~L}_{i, j}^{ \pm}(t)=k_{j}^{ \pm}(t) \mathrm{E}_{j, i}^{ \pm}(t)+\sum_{1 \leq m<j} \mathrm{~F}_{j, m}^{ \pm}(t) k_{m}^{ \pm}(t) \mathrm{E}_{m, i}^{ \pm}(t), \quad 3 \geq i>j \geq 1 .
\end{aligned}
$$

Using the arguments of [19], we may obtain for the linear combinations of the Gauss coordinates $(i=1,2)$

$$
F_{i}(t)=\mathrm{F}_{i+1, i}^{+}(t)-\mathrm{F}_{i+1, i}^{-}(t), \quad E_{i}(t)=\mathrm{E}_{i, i+1}^{+}(t)-\mathrm{E}_{i, i+1}^{-}(t),
$$

and for the Cartan currents $k_{i}^{ \pm}(t)$, the commutation relations of the quantum affine algebra $U_{q}\left(\widehat{\mathfrak{g l}}_{3}\right)$ with zero central charge and the gradation operator dropped out. In terms of the total currents $F_{i}(t), E_{i}(t)$ and of the Cartan currents $k_{i}^{ \pm}(t)$, these commutation relations read

$$
\begin{aligned}
& \left(q z-q^{-1} w\right) E_{i}(z) E_{i}(w)=E_{i}(w) E_{i}(z)\left(q^{-1} z-q w\right), \\
& \left(q^{-1} z-q w\right) E_{i}(z) E_{i+1}(w)=E_{i+1}(w) E_{i}(z)(z-w), \\
& k_{i}^{ \pm}(z) E_{i}(w)\left(k_{i}^{ \pm}(z)\right)^{-1}=\frac{z-w}{q^{-1} z-q w} E_{i}(w), \\
& k_{i+1}^{ \pm}(z) E_{i}(w)\left(k_{i+1}^{ \pm}(z)\right)^{-1}=\frac{z-w}{q z-q^{-1} w} E_{i}(w), \\
& k_{i}^{ \pm}(z) E_{j}(w)\left(k_{i}^{ \pm}(z)\right)^{-1}=E_{j}(w), \quad \text { if } \quad i \neq j, j+1, \\
& \left(q^{-1} z-q w\right) F_{i}(z) F_{i}(w)=F_{i}(w) F_{i}(z)\left(q z-q^{-1} w\right), \\
& (z-w) F_{i}(z) F_{i+1}(w)=F_{i+1}(w) F_{i}(z)\left(q^{-1} z-q w\right), \\
& k_{i}^{ \pm}(z) F_{i}(w)\left(k_{i}^{ \pm}(z)\right)^{-1}=\frac{q^{-1} z-q w}{z-w} F_{i}(w), \\
& k_{i+1}^{ \pm}(z) F_{i}(w)\left(k_{i+1}^{ \pm}(z)\right)^{-1}=\frac{q z-q^{-1} w}{z-w} F_{i}(w), \\
& k_{i}^{ \pm}(z) F_{j}(w)\left(k_{i}^{ \pm}(z)\right)^{-1}=F_{j}(w), \quad \text { if } \quad i \neq j, j+1, \\
& {\left[E_{i}(z), F_{j}(w)\right]=\delta_{i, j} \delta(z / w)\left(q-q^{-1}\right)\left(k_{i+1}^{-}(z) / k_{i}^{-}(z)-k_{i+1}^{+}(w) / k_{i}^{+}(w)\right),}
\end{aligned}
$$

with Serre relations

$$
\begin{aligned}
& \operatorname{Sym}_{z_{1}, z_{2}}\left(E_{i}\left(z_{1}\right) E_{i}\left(z_{2}\right) E_{i \pm 1}(w)-\left(q+q^{-1}\right) E_{i}\left(z_{1}\right) E_{i \pm 1}(w) E_{i}\left(z_{2}\right)\right. \\
& \left.\quad+E_{i \pm 1}(w) E_{i}\left(z_{1}\right) E_{i}\left(z_{2}\right)\right)=0
\end{aligned}
$$




$$
\begin{aligned}
& \operatorname{Sym}_{z_{1}, z_{2}}\left(F_{i}\left(z_{1}\right) F_{i}\left(z_{2}\right) F_{i \pm 1}(w)-\left(q+q^{-1}\right) F_{i}\left(z_{1}\right) F_{i \pm 1}(w) F_{i}\left(z_{2}\right)\right. \\
& \left.+F_{i \pm 1}(w) F_{i}\left(z_{1}\right) F_{i}\left(z_{2}\right)\right)=0 .
\end{aligned}
$$

Formulae (3.4)-(3.14) should be considered as formal series identities describing the infinite set of relations between modes of the currents. The symbol $\delta(z)$ entering these relations is a formal series $\sum_{n \in \mathbb{Z}} z^{n}$.

\subsection{Borel subalgebras and projections on their intersections}

We consider two types of Borel subalgebras in the algebra $U_{q}\left(\widehat{\mathfrak{g l}}_{3}\right)$. Borel subalgebras $U_{q}\left(\mathfrak{b}^{ \pm}\right) \subset$ $U_{q}\left(\widehat{\mathfrak{g l}}_{3}\right)$ are generated by the modes of the L-operators $\mathrm{L}^{( \pm)}(z)$ respectively. For the generators in these subalgebras, we can use instead modes of the Gauss coordinates (3.1)-(3.3), $\mathrm{E}_{i, i+1}^{ \pm}(t)$, $\mathrm{F}_{i+1, i}^{ \pm}(t), k_{j}^{ \pm}(t)$.

Other types of Borel subalgebras are related to the current realizations of $U_{q}\left(\widehat{\mathfrak{g l}}_{N}\right)$ given in the previous subsection. We consider first the current Borel subalgebras generated by the modes of the currents $E_{i}(t), F_{i}(t), k_{j}^{ \pm}(t)$.

The Borel subalgebra $U_{F} \subset U_{q}\left(\widehat{\mathfrak{g l}}_{3}\right)$ is generated by modes of the currents $F_{i}[n], k_{j}^{+}[m]$, $i=1,2, j=1,2,3, n \in \mathbb{Z}$ and $m \geq 0$. The Borel subalgebra $U_{E} \subset U_{q}\left(\widehat{\mathfrak{g l}}_{3}\right)$ is generated by modes of the currents $E_{i}[n], k_{j}^{-}[-m], i=1,2, j=1,2,3, n \in \mathbb{Z}$ and $m \geq 0$. We will consider also the subalgebra $U_{F}^{\prime} \subset U_{F}$, generated by the elements $F_{i}[n], k_{j}^{+}[m], i=1,2, j=1,2,3, n \in \mathbb{Z}$ and $m>0$, and the subalgebra $U_{E}^{\prime} \subset U_{E}$ generated by the elements $E_{i}[n], k_{j}^{-}[-m], i=1,2$, $j=1,2,3, n \in \mathbb{Z}$ and $m>0$. In the following, we will be interested in the intersections

$$
\begin{array}{ll}
U_{f}^{-}=U_{F}^{\prime} \cap U_{q}\left(\mathfrak{b}^{-}\right), & U_{F}^{+}=U_{F} \cap U_{q}\left(\mathfrak{b}^{+}\right), \\
U_{E}^{-}=U_{E} \cap U_{q}\left(\mathfrak{b}^{-}\right), & U_{e}^{+}=U_{E}^{\prime} \cap U_{q}\left(\mathfrak{b}^{+}\right),
\end{array}
$$

and will describe properties of the projections on these intersections. We call $U_{F}$ and $U_{E}$ the current Borel subalgebras.

In [18] the current Hopf structure for the algebra $U_{q}\left(\widehat{\mathfrak{g l}}_{3}\right)$ has been defined as:

$$
\begin{aligned}
& \Delta^{(D)}\left(E_{i}(z)\right)=1 \otimes E_{i}(z)+E_{i}(z) \otimes k_{i+1}^{-}(z)\left(k_{i}^{-}(z)\right)^{-1}, \\
& \Delta^{(D)}\left(F_{i}(z)\right)=F_{i}(z) \otimes 1+k_{i+1}^{+}(z)\left(k_{i}^{+}(z)\right)^{-1} \otimes F_{i}(z), \\
& \Delta^{(D)}\left(k_{i}^{ \pm}(z)\right)=k_{i}^{ \pm}(z) \otimes k_{i}^{ \pm}(z) .
\end{aligned}
$$

With respect to the current Hopf structure, the current Borel subalgebras are Hopf subalgebras of $U_{q}\left(\widehat{\mathfrak{g l}}_{3}\right)$. One may construct the whole algebra $U_{q}\left(\widehat{\mathfrak{g l}}_{3}\right)$ from one of its current Borel subalgebras using the current Hopf structure and the Hopf pairing

$$
\begin{aligned}
& \left\langle E_{i}(z), F_{j}(w)\right\rangle=\left(q-q^{-1}\right) \delta_{i j} \delta(z / w), \\
& \left\langle\psi_{i}^{-}(z), k_{i+1}^{+}(w)\right\rangle=\left\langle k_{i}^{-}(z), \psi_{i}^{+}(w)\right\rangle^{-1}=\frac{q-q^{-1} z / w}{1-z / w}, \\
& \left\langle\psi_{i}^{-}(z), k_{i}^{+}(w)\right\rangle=\left\langle k_{i+1}^{-}(z), \psi_{i}^{+}(w)\right\rangle^{-1}=\frac{q^{-1}-q z / w}{1-z / w},
\end{aligned}
$$

where

$$
\psi_{i}^{ \pm}(t)=k_{i+1}^{ \pm}(t)\left(k_{i}^{ \pm}(t)\right)^{-1}, \quad i=1,2 .
$$


Formulas (3.16) can be obtained from the commutation relations (3.4)-(3.14) using the commutator rules (5.4) in the quantum double.

One can check $[26,27]$ that the intersections $U_{f}^{-}$and $U_{F}^{+}$, respectively $U_{e}^{+}$and $U_{E}^{-}$, are subalgebras and coideals with respect to the Drinfeld coproduct (3.15):

$$
\begin{array}{ll}
\Delta^{(D)}\left(U_{F}^{+}\right) \subset U_{F}^{+} \otimes U_{q}\left(\widehat{\mathfrak{g l}}_{3}\right), & \Delta^{(D)}\left(U_{f}^{-}\right) \subset U_{q}\left(\widehat{\mathfrak{g l}}_{3}\right) \otimes U_{f}^{-}, \\
\Delta^{(D)}\left(U_{e}^{+}\right) \subset U_{e}^{+} \otimes U_{q}\left(\widehat{\mathfrak{g l}}_{3}\right), & \Delta^{(D)}\left(U_{E}^{-}\right) \subset U_{q}\left(\widehat{\mathfrak{g l}}_{3}\right) \otimes U_{E}^{-},
\end{array}
$$

and that the multiplication $m$ in $U_{q}\left(\widehat{\mathfrak{g l}}_{3}\right)$ induces an isomorphism of vector spaces

$$
m: U_{f}^{-} \otimes U_{F}^{+} \rightarrow U_{F}, \quad m: U_{e}^{+} \otimes U_{E}^{-} \rightarrow U_{E}
$$

According to the general theory presented in [26], we introduce the projection operators

$$
\begin{aligned}
P_{f}^{+}: U_{F} \subset U_{q}\left(\widehat{\mathfrak{g l}}_{3}\right) \rightarrow U_{F}^{+}, & P_{f}^{-}: U_{F} \subset U_{q}\left(\widehat{\mathfrak{g l}}_{3}\right) \rightarrow U_{f}^{-}, \\
P_{e}^{+}: U_{E} \subset U_{q}\left(\widehat{\mathfrak{g l}}_{3}\right) \rightarrow U_{e}^{+}, & P_{e}^{-}: U_{E} \subset U_{q}\left(\widehat{\mathfrak{g l}}_{3}\right) \rightarrow U_{E}^{-} .
\end{aligned}
$$

They are respectively defined by the prescriptions

$$
\begin{array}{llll}
P_{f}^{+}\left(f_{-} f_{+}\right)=\varepsilon\left(f_{-}\right) f_{+}, & P_{f}^{-}\left(f_{-} f_{+}\right)=f_{-} \varepsilon\left(f_{+}\right), & \forall f_{-} \in U_{f}^{-}, & \forall f_{+} \in U_{F}^{+}, \\
P_{e}^{+}\left(e_{+} e_{-}\right)=e_{+} \varepsilon\left(e_{-}\right), & P_{e}^{-}\left(e_{-} e_{+}\right)=\varepsilon\left(e_{+}\right) e_{-}, \quad \forall e_{-} \in U_{E}^{-}, \quad \forall e_{+} \in U_{e}^{+},
\end{array}
$$

where $\varepsilon: U_{q}\left(\widehat{\mathfrak{g l}}_{3}\right) \rightarrow \mathbb{C}$ is a counit map.

It was proved in [28] that the projections $P_{f}^{ \pm}$and $P_{e}^{ \pm}$are adjoint with respect to the Hopf pairing (3.16)

$$
\left\langle e, P_{f}^{ \pm}(f)\right\rangle=\left\langle P_{e}^{\mp}(e), f\right\rangle .
$$

Denote by $\bar{U}_{F}$ an extension of the algebra $U_{F}$ formed by infinite sums of monomials that are ordered products $a_{i_{1}}\left[n_{1}\right] \cdots a_{i_{k}}\left[n_{k}\right]$ with $n_{1} \leq \cdots \leq n_{k}$, where $a_{i_{l}}\left[n_{l}\right]$ is either $F_{i_{l}}\left[n_{l}\right]$ or $k_{i_{l}}^{+}\left[n_{l}\right]$. Denote by $\bar{U}_{E}$ an extension of the algebra $U_{E}$ formed by infinite sums of monomials that are ordered products $a_{i_{1}}\left[n_{1}\right] \cdots a_{i_{k}}\left[n_{k}\right]$ with $n_{1} \geq \cdots \geq n_{k}$, where $a_{i_{l}}\left[n_{l}\right]$ is either $E_{i_{l}}\left[n_{l}\right]$ or $k_{i_{l}}^{-}\left[n_{l}\right]$. It was proved in $[26]$ that

(1) the action of the projections (3.18) can be extended to the algebra $\bar{U}_{F}$;

(2) for any $f \in \bar{U}_{F}$ with $\Delta^{(D)}(f)=\sum_{i} f_{i}^{\prime} \otimes f_{i}^{\prime \prime}$ we have $f=\sum_{i} P_{f}^{-}\left(f_{i}^{\prime}\right) \cdot P_{f}^{+}\left(f_{i}^{\prime \prime}\right)$;

(3) the action of the projections (3.19) can be extended to the algebra $\bar{U}_{E}$;

(4) for any $e \in \bar{U}_{E}$ with $\Delta^{(D)}(e)=\sum_{i} e_{i}^{\prime} \otimes e_{i}^{\prime \prime}$ we have $e=\sum_{i} P_{e}^{+}\left(e_{i}^{\prime \prime}\right) \cdot P_{e}^{-}\left(e_{i}^{\prime}\right)$.

\subsection{Definition of the composed currents}

We introduce the composed currents ${ }^{2} E_{1,3}(w)$ and $F_{3,1}(y)$ which are defined by the formulas

$$
\begin{aligned}
& E_{1,3}(w)=\oint_{C_{0}} \frac{d z}{z} E_{2}(z) E_{1}(w)-\oint_{C_{\infty}} \frac{d z}{z} E_{1}(w) E_{2}(z) \frac{q-q^{-1} w / z}{1-w / z} \\
& F_{3,1}(y)=\oint_{C_{0}} \frac{d v}{v} F_{1}(y) F_{2}(v)-\oint_{C_{\infty}} \frac{d v}{v} F_{2}(v) F_{1}(y) \frac{q-q^{-1} y / v}{1-y / v}
\end{aligned}
$$

\footnotetext{
${ }^{2}$ Different definitions exist for composed currents, we choose the one, such that the corresponding projections of them coincide with the Gauss coordinates (3.1).
} 
where the contour integrals $\oint_{C_{0, \infty}} \frac{d z}{z} g(z)$ are considered as integrals around zero and infinity points respectively. The composed currents $E_{1,3}(w)$ and $F_{3,1}(y)$ belong to the completed algebras $\bar{U}_{E}$ and $\bar{U}_{F}$, respectively. Let us remind that, according to these completions, we have to understand the product of currents $E_{2}(z) E_{1}(w)$ as an analytical 'function' without singularities in the domain $|z| \ll|w|$. Analogously, the product $F_{1}(y) F_{2}(v)$ is an analytical 'function' in the domain $|y| \gg|v|$. For practical calculation, the contour integrals in definitions (3.20) can be understood as the formal integrals of a Laurent series $g(z)=\sum_{k \in \mathbb{Z}} g[k] z^{-k}$ picking up its zero mode coefficient $g[0]$.

Deforming contours in the defining formulas for the composed currents we may rewrite them differently

$$
E_{1,3}(w)=-\underset{z=w}{\operatorname{res}} E_{2}(z) E_{1}(w) \frac{d z}{z}, \quad F_{3,1}(y)=-\underset{v=y}{\operatorname{res}} F_{1}(y) F_{2}(v) \frac{d v}{v},
$$

or

$$
\begin{aligned}
& E_{2}(z) E_{1}(w)=\frac{q-q^{-1} w / z}{1-w / z} E_{1}(w) E_{2}(z)+\delta(w / z) E_{1,3}(w), \\
& F_{1}(y) F_{2}(v)=\frac{q-q^{-1} y / v}{1-y / v} F_{2}(v) F_{1}(y)+\delta(y / v) F_{3,1}(y) .
\end{aligned}
$$

Formulas (3.21) are convenient for the presentation of the composed currents as products of simple root currents

$$
E_{1,3}(w)=\left(q^{-1}-q\right) E_{1}(w) E_{2}(w), \quad F_{3,1}(y)=\left(q^{-1}-q\right) F_{2}(y) F_{1}(y) .
$$

Formulas (3.22) are convenient to calculate the commutation relation between total and halfcurrents. This will be done lately.

First, we calculate the formal integrals in the formulas (3.20) to obtain

$$
\begin{aligned}
& E_{1,3}(w)=E_{2}[0] E_{1}(w)-q^{-1} E_{1}(w) E_{2}[0]-\left(q-q^{-1}\right) \sum_{k=0}^{\infty} E_{1}(w) E_{2}[-k] w^{k}, \\
& F_{3,1}(y)=F_{1}(y) F_{2}[0]-q F_{2}[0] F_{1}(y)-\left(q-q^{-1}\right) \sum_{k=1}^{\infty} F_{2}[-k] F_{1}(y) y^{k}
\end{aligned}
$$

Here we used the series expansion

$$
\frac{q-q^{-1} w / z}{1-w / z}=q+\left(q-q^{-1}\right) \sum_{k=1}^{\infty}(w / z)^{k}=q^{-1}+\left(q-q^{-1}\right) \sum_{k=0}^{\infty}(w / z)^{k} .
$$

Introducing now the half-currents

$$
E_{2}^{ \pm}(w)= \pm \sum_{\substack{k>0 \\ k \leq 0}} E_{2}[k] w^{-k}, \quad F_{2}^{ \pm}(w)= \pm \sum_{\substack{k \geq 0 \\ k<0}} F_{2}[k] w^{-k}
$$

and using the decomposition of the algebra $U_{q}\left(\widehat{\mathfrak{g l}}_{3}\right)$ into its standard positive and negative Borel subalgebras and the definition of the screening operators

$$
\begin{aligned}
& \mathrm{E}_{2}\left(E_{1}(w)\right)=E_{2}[0] E_{1}(w)-q^{-1} E_{1}(w) E_{2}[0], \\
& \mathrm{F}_{2}\left(F_{1}(y)\right)=F_{1}(y) F_{2}[0]-q F_{2}[0] F_{1}(y)
\end{aligned}
$$


we may write

$$
\begin{aligned}
& \mathrm{E}_{2}\left(E_{1}(w)\right)=E_{1,3}(w)+\left(q^{-1}-q\right) E_{1}(w) E_{2}^{-}(w), \\
& \mathrm{F}_{2}\left(F_{1}(y)\right)=F_{3,1}(y)+\left(q^{-1}-q\right) F_{2}^{-}(y) F_{1}(y),
\end{aligned}
$$

or

$$
\mathrm{E}_{2}\left(E_{1}(w)\right)=\left(q^{-1}-q\right) E_{1}(w) E_{2}^{+}(w), \quad \mathrm{F}_{2}\left(F_{1}(y)\right)=\left(q^{-1}-q\right) F_{2}^{+}(y) F_{1}(y)
$$

To obtain the latter relation, we used formulas (3.23) and relation between total and half-currents $E_{2}(w)=E_{2}^{+}(w)-E_{2}^{-}(w)$ and $F_{2}(w)=F_{2}^{+}(w)-F_{2}^{-}(w)$.

\section{Universal Bethe vectors and projections}

The goal of this section is to obtain the representations for the left and right universal Bethe vectors in terms of the integral transform of the products of the total currents. This will generalize the results obtained for $U_{q}\left(\widehat{\mathfrak{s} l_{3}}\right)$ in the paper [28]. The calculation of the scalar product after that will be reduced to the calculation of the exchange relations between products of total currents.

\subsection{Universal Bethe vectors through currents}

It was shown in the papers $[20,24]$ that the universal right Bethe vectors

$$
\mathbb{B}_{V}(\bar{t}, \bar{s})=P_{f}^{+}\left(F_{1}\left(t_{1}\right) \cdots F_{1}\left(t_{a}\right) F_{2}\left(s_{1}\right) \cdots F_{2}\left(s_{b}\right)\right) \prod_{i=1}^{a} k_{1}\left(t_{i}\right) \prod_{i=1}^{b} k_{2}\left(s_{i}\right) v
$$

can be identified with some projection of products of total currents. Using the same method, we may prove that the left Bethe vectors

$$
\mathbb{C}_{V^{\prime}}(\bar{\tau}, \bar{\sigma})=v^{\prime} \prod_{i=1}^{a} k_{1}\left(\tau_{i}\right) \prod_{i=1}^{b} k_{2}\left(\sigma_{i}\right) P_{e}^{+}\left(E_{2}\left(\sigma_{b}\right) \cdots E_{2}\left(\sigma_{1}\right) E_{1}\left(\tau_{a}\right) \cdots E_{1}\left(\tau_{1}\right)\right)
$$

can be also identified with projection of products of total currents ${ }^{3}$. So the problem of calculating the scalar product $\left\langle\mathbb{C}_{V^{\prime}}(\bar{\tau}, \bar{\sigma}), \mathbb{B}_{V}(\bar{t}, \bar{s})\right\rangle$ is reduced to the exchange relations between projections. Fortunately, to perform this exchange, we have to calculate only modulo the ideals in the algebra $U_{q}\left(\widehat{\mathfrak{g l}}_{3}\right)$ which are annihilated by the left/right singular vectors.

One could calculate these projections to present them in the form of a sum of products of projections of simple and composed root currents (see formulas (4.4) and (4.5) below). However, this calculation has the same level of difficulty as the exchange relations of Bethe vectors in terms of L-operators. The idea of the present paper is to rewrite projection formulas (4.4) and (4.5) in terms of integrals of total simple root currents, and then to compute the exchange of products of total currents. In this way, we will obtain an integral representation for the scalar product of the off-shell Bethe vectors. This calculation is much more easy, since the commutation relations of the simple roots total currents are rather simple.

\footnotetext{
${ }^{3}$ In (4.1) all operators are acting to the left onto left singular vector $v^{\prime}$.
} 


\subsection{Calculation of the universal off-shell Bethe vectors}

Before presenting the formulas for the universal off-shell Bethe vectors in terms of the current generators, we have to introduce the following notations. Consider the permutation group $S_{n}$ and its action on the formal series of $n$ variables defined, for the elementary transpositions $\sigma_{i, i+1}$, as follows

$$
\pi\left(\sigma_{i, i+1}\right) G\left(t_{1}, \ldots, t_{i}, t_{i+1}, \ldots, t_{n}\right)=\frac{q^{-1}-q t_{i} / t_{i+1}}{q-q^{-1} t_{i} / t_{i+1}} G\left(t_{1}, \ldots, t_{i+1}, t_{i}, \ldots, t_{n}\right) .
$$

The $q$-depending factor in this formula is chosen in such a way that each product $F_{a}\left(t_{1}\right) \cdots$ $F_{a}\left(t_{n}\right)$ is invariant under this action. Summing the action over all the group of permutations, we obtain the operator $\overline{\operatorname{Sym}}_{\bar{t}}=\sum_{\sigma \in S_{n}} \pi(\sigma)$ acting as follows

$$
\overline{\operatorname{Sym}}_{\bar{t}} G(\bar{t})=\sum_{\sigma \in S_{n}} \prod_{\substack{\ell<\ell^{\prime} \\ \sigma(\ell)>\sigma\left(\ell^{\prime}\right)}} \frac{q^{-1}-q t_{\sigma\left(\ell^{\prime}\right)} / t_{\sigma(\ell)}}{q-q^{-1} t_{\sigma\left(\ell^{\prime}\right)} / t_{\sigma(\ell)}} G\left({ }^{\sigma} t\right) .
$$

The product is taken over all pairs $\left(\ell, \ell^{\prime}\right)$, such that conditions $\ell<\ell^{\prime}$ and $\sigma(\ell)>\sigma\left(\ell^{\prime}\right)$ are satisfied simultaneously.

According to the results of the papers [26, 27], the calculation of the universal off-shell Bethe vectors is reduced to the calculation of the projections

$$
P_{f}^{+}\left(F_{1}\left(t_{1}\right) \cdots F_{1}\left(t_{a}\right) F_{2}\left(s_{1}\right) \cdots F_{2}\left(s_{b}\right)\right)
$$

for the right Bethe vectors and ${ }^{4}$

$$
P_{e}^{+}\left(E_{2}\left(\sigma_{b}\right) \cdots E_{2}\left(\sigma_{1}\right) E_{1}\left(\tau_{a}\right) \cdots E_{1}\left(\tau_{1}\right)\right)
$$

for the left Bethe vectors. The calculation was detailed in [28]. Here, we present the result of calculations and give several comments on how it was performed.

Proposition 1. The projections (4.2) and (4.3) are given by the series

$$
\begin{aligned}
P_{f}^{+} & \left(F_{1}\left(t_{1}\right) \cdots F_{1}\left(t_{a}\right) F_{2}\left(s_{1}\right) \cdots F_{2}\left(s_{b}\right)\right) \\
= & \sum_{k=0}^{\min \{a, b\}} \frac{1}{k !(a-k) !(b-k) !} \overline{\operatorname{Sym}}_{\bar{t}, \bar{s}}\left(P_{f}^{+}\left(F_{1}\left(t_{1}\right) \cdots F_{1}\left(t_{a-k}\right) F_{3,1}\left(t_{a-k+1}\right) \cdots F_{3,1}\left(t_{a}\right)\right)\right. \\
& \left.\times P_{f}^{+}\left(F_{2}\left(s_{k+1}\right) \cdots F_{2}\left(s_{b}\right)\right) Z\left(t_{a}, \ldots, t_{a-k+1} ; s_{k}, \ldots, s_{1}\right)\right)
\end{aligned}
$$

$a n d^{5}$

$$
\begin{aligned}
P_{e}^{+} & \left(E_{2}\left(\sigma_{b}\right) \cdots E_{2}\left(\sigma_{1}\right) E_{1}\left(\tau_{a}\right) \cdots E_{1}\left(\tau_{1}\right)\right) \\
= & \sum_{m=0}^{\min \{a, b\}} \frac{1}{m !(a-m) !(b-m) !} \overline{\operatorname{Sym}}_{\bar{\tau}, \bar{\sigma}}\left(P_{e}^{+}\left(E_{2}\left(\sigma_{b}\right) \cdots E_{2}\left(\sigma_{m+1}\right)\right)\right. \\
& \left.\times P_{e}^{+}\left(E_{1,3}\left(\tau_{a}\right) \cdots E_{1,3}\left(\tau_{a-m+1}\right) E_{1}\left(\tau_{a-m}\right) \cdots E_{1}\left(\tau_{1}\right)\right) Y\left(\tau_{a}, \ldots, \tau_{a-m+1} ; \sigma_{m}, \ldots, \sigma_{1}\right)\right),
\end{aligned}
$$

\footnotetext{
${ }^{4}$ For further convenience, we will denote the spectral parameters of the right Bethe vectors by latin symbols, and those of the left vectors by greek ones.

${ }^{5}$ The ordering of the variables in the rational series in (4.4) differs from the ordering in the corresponding series in the paper [28]. This is because the rational series (4.6) are defined differently with respect to the paper [28].
} 
where

$$
\begin{aligned}
Y\left(t_{1}, \ldots, t_{n} ; x_{1}, \ldots, x_{n}\right) & =\prod_{i=1}^{n} \frac{1}{1-x_{i} / t_{i}} \prod_{j=1}^{i-1} \frac{q^{-1}-q x_{i} / t_{j}}{1-x_{i} / t_{j}} \\
& =\prod_{i=1}^{n} \frac{1}{1-x_{i} / t_{i}} \prod_{j=i+1}^{n} \frac{q^{-1}-q x_{j} / t_{i}}{1-x_{j} / t_{i}} \\
Z\left(t_{1}, \ldots, t_{n} ; x_{1}, \ldots, x_{n}\right) & =Y\left(t_{1}, \ldots, t_{n} ; x_{1}, \ldots, x_{n}\right) \prod_{i=1}^{n} \frac{x_{i}}{t_{i}}
\end{aligned}
$$

Note that the kernels (4.6) are defined in such a way, that they have only $k$ simple poles at the point $t_{1}, \ldots, t_{k}$ with respect to the variable $x_{k}, k=1, \ldots, n$. These kernels appear in the integral presentation of the projections of the products of the same simple root currents (see (4.14) below).

The proof of the formulas (4.4) and (4.5) is similar to the proof presented in the paper [28]. We will not repeat this calculations here, but for completeness, we collect all necessary formulas. As a first step, we present the products of currents $F_{2}\left(s_{1}\right) \cdots F_{2}\left(s_{b}\right)$ and $E_{2}\left(\sigma_{b}\right) \cdots E_{2}\left(\sigma_{1}\right)$ in a normal ordered form using properties of the projections given at the end of the Subsection 3.2:

$$
\begin{aligned}
& F_{2}\left(s_{1}\right) \cdots F_{2}\left(s_{b}\right)=\sum_{k=0}^{b} \frac{1}{k !(b-k) !} \overline{\operatorname{Sym}}_{\bar{s}}\left(P_{f}^{-}\left(F_{2}\left(s_{1}\right) \cdots F_{2}\left(s_{k}\right)\right) \cdot P_{f}^{+}\left(F_{2}\left(s_{k+1}\right) \cdots F_{2}\left(s_{b}\right)\right)\right), \\
& E_{2}\left(\sigma_{b}\right) \cdots E_{2}\left(\sigma_{1}\right) \\
& =\sum_{m=0}^{b} \frac{1}{m !(b-m) !} \overline{\operatorname{Sym}}_{\bar{\sigma}}\left(P_{e}^{+}\left(E_{2}\left(\sigma_{b}\right) \cdots E_{2}\left(\sigma_{m+1}\right)\right) \cdot P_{e}^{-}\left(E_{2}\left(\sigma_{m}\right) \cdots E_{2}\left(\sigma_{1}\right)\right)\right) .
\end{aligned}
$$

To evaluate the projections in formulas (4.4) and (4.5), we commute the negative projections $P_{f}^{-}\left(F_{2}\left(s_{1}\right) \cdots F_{2}\left(s_{k}\right)\right)$ to the left through the product of the total currents $F_{1}\left(t_{1}\right) \cdots F_{1}\left(t_{a}\right)$ in case of (4.4) and commute the negative projections $P_{e}^{-}\left(E_{2}\left(s_{m}\right) \cdots E_{2}\left(s_{1}\right)\right)$ to the right through the product of the total currents $E_{1}\left(\tau_{a}\right) \cdots E_{1}\left(\tau_{1}\right)$ in (4.5). To perform this commutation we use

$$
\begin{aligned}
& P_{f}^{-}\left(F_{2}\left(s_{1}\right) \cdots F_{2}\left(s_{k}\right)\right)=(-1)^{k} F_{2}^{-}\left(s_{1} ; s_{2}, \ldots, s_{k}\right) \cdots F_{2}^{-}\left(s_{k-1} ; s_{k}\right) F_{2}^{-}\left(s_{k}\right), \\
& P_{e}^{-}\left(E_{2}\left(\sigma_{m}\right) \cdots E_{2}\left(\sigma_{1}\right)\right)=(-1)^{m} E_{2}^{-}\left(\sigma_{m}\right) E_{2}^{-}\left(\sigma_{m-1} ; \sigma_{m}\right) \cdots E_{2}^{-}\left(\sigma_{1} ; \sigma_{2}, \ldots, \sigma_{m}\right),
\end{aligned}
$$

and

$$
\begin{aligned}
& F_{3,1}(t) F_{2}^{-}\left(s_{1} ; s_{2}, \ldots, s_{k}\right)=\frac{q^{-1} s_{1}-q t}{s_{1}-t} F_{2}^{-}\left(s_{1} ; s_{2}, \ldots, s_{k}, t\right) F_{3,1}(t), \\
& E_{2}^{-}\left(\sigma_{1} ; \sigma_{2}, \ldots, \sigma_{m}\right) E_{1,3}(\tau)=\frac{q^{-1} \sigma_{1}-q \tau}{\sigma_{1}-\tau} E_{1,3}(\tau) E_{2}^{-}\left(\sigma_{1} ; \sigma_{2}, \ldots, \sigma_{m}, \tau\right) .
\end{aligned}
$$

The expressions

$$
\begin{aligned}
& F_{2}^{-}\left(s_{1} ; s_{2}, \ldots, s_{k}\right)=F_{2}^{-}\left(s_{1}\right)-\sum_{\ell=2}^{k} \frac{s_{1}}{s_{\ell}} \phi_{s_{\ell}}\left(s_{1} ; s_{2}, \ldots, s_{k}\right) F_{2}^{-}\left(s_{\ell}\right), \\
& E_{2}^{-}\left(\sigma_{1} ; \sigma_{2}, \ldots, \sigma_{m}\right)=E_{2}^{-}\left(\sigma_{1}\right)-\sum_{\ell=2}^{m} \phi_{\sigma_{\ell}}\left(\sigma_{1} ; \sigma_{2}, \ldots, \sigma_{m}\right) E_{2}^{-}\left(\sigma_{\ell}\right)
\end{aligned}
$$

are linear combinations of the half-currents, while

$$
\phi_{s_{\ell}}\left(s_{1} ; s_{2}, \ldots, s_{k}\right)=\prod_{j=2, j \neq \ell}^{k} \frac{s_{1}-s_{j}}{s_{\ell}-s_{j}} \prod_{j=2}^{k} \frac{q^{-1} s_{\ell}-q s_{j}}{q^{-1} s_{1}-q s_{j}}
$$


are rational functions satisfying the normalization conditions $\phi_{s_{j}}\left(s_{i} ; s_{2}, \ldots, s_{k}\right)=\delta_{i j}, i, j=$ $2, \ldots, k$. One also needs the commutation relations between negative half-currents and the total currents

$$
\begin{aligned}
& F_{1}(t) F_{2}^{-}(s)=\frac{q s-q^{-1} t}{s-t}\left(F_{2}^{-}(s)-\frac{\left(q-q^{-1}\right) s}{q s-q^{-1} t} F_{2}^{-}(t)\right) F_{1}(t)+\frac{s}{t-s} F_{3,1}(t), \\
& E_{2}^{-}(\sigma) E_{1}(\tau)=\frac{q \sigma-q^{-1} \tau}{\sigma-\tau} E_{1}(\tau)\left(E_{2}^{-}(\sigma)-\frac{\left(q-q^{-1}\right) \tau}{q \sigma-q^{-1} \tau} E_{2}^{-}(\tau)\right)+\frac{\tau}{\sigma-\tau} E_{1,3}(\tau),
\end{aligned}
$$

and the identity

$$
\prod_{i<j} \frac{q^{-1} t_{i}-q t_{j}}{t_{i}-t_{j}} \overline{\operatorname{Sym}}_{\bar{t}}\left(Y\left(t_{n}, \ldots, t_{1} ;{ }^{\omega} \bar{s}\right)\right)=\prod_{i<j} \frac{q^{-1} s_{i}-q s_{j}}{s_{i}-s_{j}} \overline{\operatorname{Sym}}_{\bar{s}}\left(Y\left({ }^{\prime} \bar{t}_{j} s_{n}, \ldots, s_{1}\right)\right)
$$

valid for arbitrary permutations $\omega$ and $\omega^{\prime}$ of the sets $\bar{s}$ and $\bar{t}$, respectively.

\subsection{Integral presentation of the projections (4.4) and (4.5)}

The projections (4.4) and (4.5) are given as a product of projection of currents. As already mentioned, this form is not convenient to obtain scalar products. We give a new representation in term of a multiple integral over the product of simple root currents:

\section{Proposition 2.}

$$
\begin{aligned}
P_{e}^{+} & \left(E_{2}\left(\sigma_{b}\right) \cdots E_{2}\left(\sigma_{1}\right) E_{1}\left(\tau_{a}\right) \cdots E_{1}\left(\tau_{1}\right)\right) \\
& =\oint \frac{d \nu_{1}}{\nu_{1}} \cdots \oint \frac{d \nu_{b}}{\nu_{b}} \oint \frac{d \mu_{1}}{\mu_{1}} \cdots \oint \frac{d \mu_{a}}{\mu_{a}} \mathbb{E}(\bar{\tau}, \bar{\sigma} ; \bar{\mu}, \bar{\nu}) E_{1}\left(\mu_{1}\right) \cdots E_{1}\left(\mu_{a}\right) E_{2}\left(\nu_{b}\right) \cdots E_{2}\left(\nu_{1}\right), \\
P_{f}^{+} & \left(F_{1}\left(t_{1}\right) \cdots F_{1}\left(t_{a}\right) F_{2}\left(s_{1}\right) \cdots F_{2}\left(s_{b}\right)\right) \\
& =\oint \frac{d y_{1}}{y_{1}} \cdots \oint \frac{d y_{b}}{y_{b}} \oint \frac{d x_{1}}{x_{1}} \cdots \oint \frac{d x_{a}}{x_{a}} \mathbb{F}(\bar{t}, \bar{s} ; \bar{x}, \bar{y}) F_{2}\left(y_{1}\right) \cdots F_{2}\left(y_{b}\right) F_{1}\left(x_{a}\right) \cdots F_{1}\left(x_{1}\right),
\end{aligned}
$$

where the kernels $\mathbb{E}(\bar{\tau}, \bar{\sigma} ; \bar{\mu}, \bar{\nu})$ and $\mathbb{F}(\bar{t}, \bar{s} ; \bar{x}, \bar{y})$ are given by the series

$$
\begin{aligned}
& \mathbb{E}(\bar{\tau}, \bar{\sigma} ; \bar{\mu}, \bar{\nu})=\overline{\operatorname{Sym}}_{\bar{\tau}, \bar{\sigma}}\left(\sum_{m=0}^{\min \{a, b\}} \frac{\left(q^{-1}-q\right)^{m}}{m !(a-m) !(b-m) !} \prod_{m<i<j \leq b} \frac{\sigma_{i}-\sigma_{j}}{q^{-1} \sigma_{i}-q \sigma_{j}}\right. \\
& \quad \times \prod_{\substack{1 \leq i<j \leq a-m \\
a-m<i<j \leq a}} \frac{\tau_{i}-\tau_{j}}{q^{-1} \tau_{i}-q \tau_{j}} Y\left(\tau_{a}, \ldots, \tau_{a-m+1} ; \sigma_{m}, \ldots, \sigma_{1}\right) Z\left(\tau_{a}, \ldots, \tau_{1} ; \mu_{a}, \ldots, \mu_{1}\right) \\
& \left.\quad \times Z\left(\mu_{a}, \ldots, \mu_{a-m+1}, \sigma_{m+1}, \ldots, \sigma_{b} ; \nu_{1}, \ldots, \nu_{b}\right) \prod_{j=1}^{a-m} \prod_{i=m+1}^{b} \frac{q^{-1}-q \nu_{i} / \mu_{j}}{1-\nu_{i} / \mu_{j}}\right)
\end{aligned}
$$

and

$$
\begin{aligned}
\mathbb{F}(\bar{t}, \bar{s} ; \bar{x}, \bar{y}) & =\overline{\operatorname{Sym}}_{\bar{t}, \bar{s}}\left(\sum_{k=0}^{\min \{a, b\}} \frac{\left(q^{-1}-q\right)^{k}}{k !(a-k) !(b-k) !} \prod_{k<i<j \leq b} \frac{s_{i}-s_{j}}{q^{-1} s_{i}-q s_{j}}\right. \\
& \times \prod_{\substack{1 \leq i<j \leq a-k \\
a-k<i<j \leq a}} \frac{t_{i}-t_{j}}{q^{-1} t_{i}-q t_{j}} Z\left(t_{a}, \ldots, t_{a-k+1} ; s_{k}, \ldots, s_{1}\right) Y\left(t_{a}, \ldots, t_{1} ; x_{a}, \ldots, x_{1}\right) \\
& \left.\times Y\left(x_{a}, \ldots, x_{a-k+1}, s_{k+1}, \ldots, s_{b} ; y_{1}, \ldots, y_{b}\right) \prod_{j=1}^{a-k} \prod_{i=k+1}^{b} \frac{q^{-1}-q y_{i} / x_{j}}{1-y_{i} / x_{j}}\right) .
\end{aligned}
$$


The proof of these formulas is given in the next subsection. Let us explain the meaning of the integral formulas for the projections (4.8). There is a preferable order of integration in these formulas. First, we have to calculate the integrals over variables $\nu_{i}$ and $y_{i}, i=1, \ldots, b$, respectively, and then calculate the integrals over $\mu_{j}$ and $x_{j}, j=1, \ldots, a$.

Example 1. Let us illustrate how it works in the simplest example $a=b=1$ and for projection $P_{f}^{+}\left(F_{1}(t) F_{2}(s)\right)$. We have

$$
P_{f}^{+}\left(F_{1}(t) F_{2}(s)\right)=\oint \frac{d y}{y} \oint \frac{d x}{x} \mathbb{F}(t, s ; x, y) F_{2}(y) F_{1}(x),
$$

where

$$
\mathbb{F}(t, s ; x, y)=Y(t ; x) Y(s ; y) \frac{q^{-1}-q y / x}{1-y / x}+\left(q^{-1}-q\right) Z(t, s) Y(t ; x) Y(x ; y) .
$$

Integration over $y$ with the first term of the kernel yields to

$$
\oint \frac{d y}{y} \frac{1}{1-y / s} \frac{q^{-1}-q y / x}{1-y / x} F_{2}(y) F_{1}(x)=\frac{q^{-1} x-q s}{x-s} F_{2}^{+}(s ; x) F_{1}(x)=F_{1}(x) F_{2}^{+}(s),
$$

due to the commutation relations (4.19). Integration over $y$ with the second term of the kernel produces

$$
\left(q^{-1}-q\right) F_{2}^{+}(x) F_{1}(x)=\mathrm{F}_{2}\left(F_{1}(x)\right),
$$

according to the formulas (3.24). Finally, integration over $x$ in both terms produces the result for the projection in this simplest case. The general case can be treated analogously. Of course, one can first integrate over $x$ and then over $y$. However in this case, the calculation of the integrals for the projection becomes more involved and requires more complicated commutation relations between half-currents.

\subsection{Proof of the integral presentation of the projections (4.4) and (4.5)}

Integral representation for the projections of the same type of currents $P_{f}^{+}\left(F_{i}\left(s_{1}\right) \cdots F_{i}\left(s_{b}\right)\right)$ and $P_{e}^{+}\left(E_{i}\left(\sigma_{b}\right) \cdots E_{i}\left(\sigma_{1}\right)\right)(i=1,2)$ were obtained in [28]. They can be obtained from the calculation of these projections

$$
\begin{aligned}
& P_{f}^{+}\left(F_{i}\left(s_{1}\right) \cdots F_{i}\left(s_{b}\right)\right)=F_{i}^{+}\left(s_{1}\right) F_{i}^{+}\left(s_{2} ; s_{1}\right) \cdots F_{i}^{+}\left(s_{b} ; s_{b-1}, \ldots, s_{1}\right), \\
& P_{e}^{+}\left(E_{i}\left(\sigma_{b}\right) \cdots E_{i}\left(\sigma_{1}\right)\right)=E_{i}^{+}\left(\sigma_{b} ; \sigma_{b-1}, \ldots, \sigma_{1}\right) \cdots E_{i}^{+}\left(\sigma_{2} ; \sigma_{1}\right) E_{i}^{+}\left(\sigma_{1}\right),
\end{aligned}
$$

where $F_{i}^{+}\left(s_{k} ; s_{k-1}, \ldots, s_{1}\right)$ and $E_{i}^{+}\left(\sigma_{k} ; \sigma_{k-1}, \ldots, \sigma_{1}\right)$ are linear combinations of the half-currents

$$
\begin{aligned}
& F_{i}^{+}\left(s_{k} ; s_{k-1}, \ldots, s_{1}\right)=F_{i}^{+}\left(s_{k}\right)-\sum_{\ell=1}^{k-1} \frac{s_{k}}{s_{\ell}} \varphi_{s_{\ell}}\left(s_{k} ; s_{k-1}, \ldots, s_{1}\right) F_{i}^{+}\left(s_{\ell}\right), \\
& E_{i}^{+}\left(\sigma_{k} ; \sigma_{k-1}, \ldots, \sigma_{1}\right)=E_{i}^{+}\left(\sigma_{k}\right)-\sum_{\ell=1}^{k-1} \varphi_{\sigma_{\ell}}\left(\sigma_{k} ; \sigma_{k-1}, \ldots, \sigma_{1}\right) F_{i}^{+}\left(\sigma_{\ell}\right),
\end{aligned}
$$

with coefficients being rational functions

$$
\varphi_{s_{\ell}}\left(s_{k} ; s_{k-1}, \ldots, s_{1}\right)=\prod_{j=1, j \neq \ell}^{k-1} \frac{s_{k}-s_{j}}{s_{\ell}-s_{j}} \prod_{j=1}^{k-1} \frac{q s_{\ell}-q^{-1} s_{j}}{q s_{k}-q^{-1} s_{j}} .
$$

There is a very simple analytical proof of the formulas (4.11) given in [28]. 
Example 2. Let us illustrate this method on one example: the first relation in (4.11) with $b=2$. Indeed, from the commutation relation of the total currents $F_{i}\left(s_{1}\right)$ and $F_{i}\left(s_{2}\right)$, and due to the integral presentation of negative half-currents

$$
F_{i}^{-}\left(s_{2}\right)=-\int \frac{d y}{y} \frac{s_{2} / y}{1-s_{2} / y} F_{i}(y)
$$

we know that

$$
P_{f}^{+}\left(F_{i}\left(s_{1}\right) F_{i}\left(s_{2}\right)\right)=F_{i}^{+}\left(s_{1}\right) F_{i}^{+}\left(s_{2}\right)+\frac{s_{2}}{s_{1}} \frac{X\left(s_{1}\right)}{q^{-1} s_{1}-q s_{2}},
$$

where $X\left(s_{1}\right)$ is an unknown algebraic element which depends only on the spectral parameter $s_{1}$. This element can be uniquely defined from the relation (4.13) setting $s_{1}=s_{2}$ and using the fact that $F_{i}^{2}(s)=0$. The general case can be treated analogously (see details in [28]). Formulas (4.7) can be proved in the same way.

Using now the integral form of the half-currents

$$
F_{i}^{+}(s)=\int \frac{d y}{y} \frac{1}{1-y / s} F_{i}(y), \quad E_{i}^{+}(s)=\int \frac{d y}{y} \frac{y / s}{1-y / s} E_{i}(y)
$$

one can easily obtain integral formulas for (4.11):

$$
\begin{aligned}
& P_{f}^{+}\left(F_{i}\left(s_{1}\right) \cdots F_{i}\left(s_{b}\right)\right)=\prod_{1 \leq i<j \leq b} \frac{s_{i}-s_{j}}{q^{-1} s_{i}-q s_{j}} \\
& \quad \times \int \frac{d y_{1}}{y_{1}} \cdots \frac{d y_{b}}{y_{b}} F_{i}\left(y_{1}\right) \cdots F_{i}\left(y_{b}\right) Y\left(s_{1}, \ldots, s_{b} ; y_{1}, \ldots, y_{b}\right), \\
& P_{e}^{+}\left(E_{i}\left(\sigma_{b}\right) \cdots E_{i}\left(\sigma_{1}\right)\right)=\prod_{1 \leq i<j \leq b} \frac{\sigma_{i}-\sigma_{j}}{q^{-1} \sigma_{i}-q \sigma_{j}} \\
& \quad \times \int \frac{d \nu_{1}}{\nu_{1}} \cdots \frac{d \nu_{b}}{\nu_{b}} E_{i}\left(\nu_{b}\right) \cdots E_{i}\left(\nu_{1}\right) Z\left(\sigma_{1}, \ldots, \sigma_{b} ; \nu_{1}, \ldots, \nu_{b}\right) .
\end{aligned}
$$

According to the structure of the kernels (4.6), the integrands in (4.14) have only simple poles with respect to the integration variables $y_{1}$ and $\nu_{1}$ in the points $s_{1}$ and $\sigma_{1}$ respectively, while with respect to the variables $y_{b}$ and $\nu_{b}$ they have simple poles in the points $s_{j}$ and $\sigma_{j}, j=1, \ldots, b$. Due to $q$-symmetric prefactors in the integrals (4.14), the integrals themselves are symmetric with respect to the spectral parameters $s_{j}$ and $\sigma_{j}, j=1, \ldots, b$, respectively.

The integral form for the projections of the strings

$$
P_{f}^{+}\left(F_{1}\left(t_{1}\right) \cdots F_{1}\left(t_{a-k}\right) F_{3,1}\left(t_{a-k+1}\right) \cdots F_{3,1}\left(t_{a}\right)\right)
$$

and

$$
P_{e}^{+}\left(E_{1,3}\left(\tau_{a}\right) \cdots E_{1,3}\left(\tau_{a-m+1}\right) E_{1}\left(\tau_{a-m}\right) \cdots E_{1}\left(\tau_{1}\right)\right)
$$

is a more delicate question. To present them as integrals, we use arguments of [28] and formulas (3.24). The point is that the analytical properties of the reverse strings

$$
P_{f}^{+}\left(F_{3,1}\left(t_{a}\right) \cdots F_{3,1}\left(t_{a-k+1}\right) F_{1}\left(t_{a-k}\right) \cdots F_{1}\left(t_{1}\right)\right)
$$

and

$$
P_{e}^{+}\left(E_{1}\left(\tau_{1}\right) \cdots E_{1}\left(\tau_{a-m}\right) E_{1,3}\left(\tau_{a-m+1}\right) \cdots E_{1,3}\left(\tau_{a}\right)\right)
$$


are the same as the analytical properties of the product of the simple root currents $F_{1}\left(t_{a}\right) \cdots F_{1}\left(t_{1}\right)$ and $E_{1}\left(\tau_{1}\right) \cdots E_{1}\left(\tau_{a}\right)$. Therefore the calculation of projection of the reverse string can be done along the same steps as for the product of simple root currents. In order to relate the projection of the string and projection of the reverse string, we need the commutation relations

$$
\begin{aligned}
& F_{1}\left(t_{1}\right) F_{3,1}\left(t_{2}\right)=\frac{q t_{1}-q^{-1} t_{2}}{t_{1}-t_{2}} F_{3,1}\left(t_{2}\right) F_{1}\left(t_{1}\right), \\
& E_{1,3}\left(\tau_{2}\right) E_{1}\left(\tau_{1}\right)=\frac{q \tau_{1}-q^{-1} \tau_{2}}{\tau_{1}-\tau_{2}} E_{1}\left(\tau_{1}\right) E_{1,3}\left(\tau_{2}\right)
\end{aligned}
$$

and the fact (proved in [28]) that under projections we can freely exchange currents without taking into account the $\delta$-function terms. As result, we get

$$
\begin{aligned}
P_{f}^{+}( & \left.F_{1}\left(t_{1}\right) \cdots F_{1}\left(t_{a-k}\right) F_{3,1}\left(t_{a-k+1}\right) \cdots F_{3,1}\left(t_{a}\right)\right) \\
= & \prod_{\substack{1 \leq i \leq a-k \\
a-k<j \leq a}} \frac{q t_{i}-q^{-1} t_{j}}{t_{i}-t_{j}} \prod_{\substack{1 \leq i<j \leq a-k \\
a-k<i<j \leq a}} \frac{q t_{i}-q^{-1} t_{j}}{q^{-1} t_{i}-q t_{j}} \\
& \times \prod_{\substack{a \geq \ell>a-k \\
=}} P_{f}^{+}\left(F_{3,1}\left(t_{\ell} ; t_{\ell+1}, \ldots, t_{a}\right)\right) \prod_{a-k \geq \ell \geq 1} F_{1}^{+}\left(t_{\ell} ; t_{\ell+1}, \ldots, t_{a}\right) \\
= & \prod_{\substack{1 \leq i<j \leq a-k \\
a-k<i<j \leq a}} \frac{t_{i}-t_{j}}{q^{-1} t_{i}-q t_{j}} \int \frac{d x_{1}}{x_{1}} \cdots \frac{d x_{a}}{x_{a}} Y\left(t_{a}, \ldots, t_{1} ; x_{a}, \ldots, x_{1}\right) \\
& \times F_{2}\left(F_{1}\left(x_{a}\right)\right) \cdots F_{2}\left(F_{1}\left(x_{a-k+1}\right)\right) F_{1}\left(x_{a-k}\right) \cdots F_{1}\left(x_{1}\right) .
\end{aligned}
$$

Analogously

$$
\begin{aligned}
& P_{e}^{+}\left(E_{1,3}\left(\tau_{a}\right) \cdots E_{1,3}\left(\tau_{a-m+1}\right) E_{1}\left(\tau_{a-m}\right) \cdots E_{1}\left(\tau_{1}\right)\right) \\
& =\prod_{\substack{1 \leq i \leq a-k \\
a-k<j \leq a}} \frac{q \tau_{i}-q^{-1} \tau_{j}}{\tau_{i}-\tau_{j}} \prod_{\substack{1 \leq i<j \leq a-k \\
a-k<i<j \leq a}} \frac{q \tau_{i}-q^{-1} \tau_{j}}{q^{-1} \tau_{i}-q \tau_{j}} \\
& \times \prod_{1 \leq \ell \leq a-m} E_{1}^{+}\left(t_{\ell} ; t_{\ell+1}, \ldots, t_{a}\right) \prod_{a-k<\ell \leq a} P_{e}^{+}\left(E_{1,3}\left(t_{\ell} ; t_{\ell+1}, \ldots, t_{a}\right)\right) \\
& =\prod_{\substack{1 \leq i<j \leq a-m \\
a-m<i<j \leq a}} \frac{\tau_{i}-\tau_{j}}{q^{-1} \tau_{i}-q \tau_{j}} \int \frac{d \mu_{1}}{\mu_{1}} \cdots \frac{d \mu_{a}}{\mu_{a}} Z\left(\tau_{a}, \ldots, \tau_{1} ; \mu_{a}, \ldots, \mu_{1}\right) \\
& \times E_{1}\left(\mu_{1}\right) \cdots E_{1}\left(\mu_{a-m}\right) \mathrm{E}_{2}\left(E_{1}\left(\mu_{a-m+1}\right)\right) \cdots \mathrm{E}_{2}\left(E_{1}\left(\mu_{a}\right)\right) .
\end{aligned}
$$

Here we used the notations

$$
\overleftarrow{\prod_{a \geq \ell \geq 1}} A_{\ell}=A_{a} A_{a-1} \cdots A_{2} A_{1}, \quad \prod_{1 \leq \ell \leq a} B_{\ell}=B_{1} B_{2} \cdots B_{a-1} B_{a}
$$

for products of non-commutative terms and the identities

$$
\begin{aligned}
& P_{f}^{+}\left(F_{3,1}(t)\right)=P_{f}^{+}\left(\mathrm{F}_{2}\left(F_{1}(t)\right)\right)=\mathrm{F}_{2}\left(P_{f}^{+}\left(F_{1}(t)\right)\right)=\mathrm{F}_{2}\left(F_{1}^{+}(t)\right), \\
& P_{e}^{+}\left(E_{1,3}(\tau)\right)=P_{e}^{+}\left(\mathrm{E}_{2}\left(E_{1}(\tau)\right)\right)=\mathrm{E}_{2}\left(P_{e}^{+}\left(E_{1}(\tau)\right)\right)=\mathrm{E}_{2}\left(E_{1}^{+}(\tau)\right),
\end{aligned}
$$

on commutativity of the screening operators and the projections proved in [28].

The last step before getting integral formulas for universal Bethe vectors is to present products of screening operators acting on total currents,

$$
\mathrm{F}_{2}\left(F_{1}\left(x_{k}\right)\right) \cdots \mathrm{F}_{2}\left(F_{1}\left(x_{1}\right)\right), \quad \mathrm{E}_{2}\left(E_{1}\left(\mu_{1}\right)\right) \cdots \mathrm{E}_{2}\left(E_{1}\left(\mu_{m}\right)\right),
$$


as an integral using formulas (3.24). The presentation follows from the following chain of equalities

$$
\begin{aligned}
& \mathrm{F}_{2}\left(F_{1}\left(x_{k}\right)\right) \cdots \mathrm{F}_{2}\left(F_{1}\left(x_{1}\right)\right)=\left(q^{-1}-q\right)^{k} F_{2}^{+}\left(x_{k}\right) F_{1}\left(x_{k}\right) \cdots F_{2}^{+}\left(x_{2}\right) F_{1}\left(x_{2}\right) F_{2}^{+}\left(x_{1}\right) F_{1}\left(x_{1}\right) \\
& =\left(q^{-1}-q\right)^{k} \prod_{1 \leq i<j \leq k} \frac{q x_{i}-q^{-1} x_{j}}{x_{i}-x_{j}} F_{2}^{+}\left(x_{k}\right) F_{2}^{+}\left(x_{k-1} ; x_{k}\right) \cdots F_{2}^{+}\left(x_{1} ; x_{2}, \ldots, x_{k}\right) F_{1}\left(x_{k}\right) \cdots F_{1}\left(x_{1}\right) \\
& =\left(q^{-1}-q\right)^{k} \int \frac{d z_{1}}{z_{1}} \cdots \frac{d z_{z}}{z_{k}} Y\left(x_{k}, \ldots, x_{1} ; z_{k}, \ldots, z_{1}\right) F_{2}\left(z_{k}\right) \cdots F_{2}\left(z_{1}\right) F_{1}\left(x_{k}\right) \cdots F_{1}\left(x_{1}\right)(4.17)
\end{aligned}
$$

and

$$
\begin{aligned}
& \mathrm{E}_{2}\left(E_{1}\left(\mu_{1}\right)\right) \cdots \mathrm{E}_{2}\left(E_{1}\left(\mu_{m}\right)\right)=\left(q^{-1}-q\right)^{k} E_{1}\left(\mu_{1}\right) E_{2}^{+}\left(\mu_{1}\right) \cdots E_{1}\left(\mu_{m}\right) E_{2}^{+}\left(\mu_{m}\right) \\
& =\left(q^{-1}-q\right)^{m} \prod_{1 \leq i<j \leq m} \frac{q \mu_{i}-q^{-1} \mu_{j}}{\mu_{i}-\mu_{j}} E_{1}\left(\mu_{1}\right) \cdots E_{1}\left(\mu_{m}\right) E_{2}^{+}\left(\mu_{1} ; \mu_{2}, \ldots, \mu_{m}\right) \cdots E_{2}^{+}\left(\mu_{m}\right) \\
& =\left(q^{-1}-q\right)^{m} \int \frac{d \rho_{1}}{\rho_{1}} \cdots \frac{d \rho_{z}}{\rho_{m}} Z\left(\mu_{m}, \ldots, \mu_{1} ; \rho_{m}, \ldots, \rho_{1}\right) E_{1}\left(\mu_{1}\right) \cdots E_{1}\left(\mu_{m}\right) E_{2}\left(\rho_{1}\right) \cdots E_{2}\left(\rho_{m}\right),
\end{aligned}
$$

where we have used the commutation relation

$$
\begin{aligned}
& F_{1}\left(x_{j}\right) F_{2}^{+}\left(x_{i} ; x_{i-1}, \ldots, x_{j-1}\right)=\frac{q x_{i}-q^{-1} x_{j}}{x_{i}-x_{j}} F_{2}^{+}\left(x_{i} ; x_{i-1}, \ldots, x_{j}\right) F_{1}\left(x_{j}\right), \\
& E_{2}^{+}\left(\mu_{i} ; \mu_{i+1}, \ldots, \mu_{j-1}\right) E_{1}\left(\mu_{j}\right)=\frac{q \mu_{i}-q^{-1} \mu_{j}}{\mu_{i}-\mu_{j}} E_{1}\left(\mu_{j}\right) E_{2}^{+}\left(\mu_{i} ; \mu_{i+1}, \ldots, \mu_{j}\right) .
\end{aligned}
$$

Note that these commutation formulas are crucial for the integral formulas given below in (4.20) and (4.21). One can see that the right hand sides of these formulas are not ordered, while the left hand sides are.

Example 3. Let us check the first equality in (4.19), in the simplest case. To calculate this exchange relation, we start from the definition of the composed currents $F_{3,1}(x)$ as given in (3.22) and apply to this relation the integral transformation

$$
\int \frac{d y}{y} \frac{1}{1-y / x_{1}}
$$

To calculate this integral, we decompose the kernel of the integrand as

$$
\frac{q-q^{-1} x_{2} / y}{1-x_{2} / y} \cdot \frac{1}{1-y / x_{1}}=\frac{q-q^{-1} x_{2} / x_{1}}{1-x_{2} / x_{1}} \cdot \frac{1}{1-y / x_{1}}+\frac{\left(q-q^{-1}\right)}{1-x_{2} / x_{1}} \cdot \frac{x_{2} / y}{1-x_{2} / y} .
$$

This leads to

$$
\begin{aligned}
F_{1}\left(x_{2}\right) F_{2}^{+}\left(x_{1}\right)= & \frac{q-q^{-1} x_{2} / x_{1}}{1-x_{2} / x_{1}} F_{2}^{+}\left(x_{1}\right) F_{1}\left(x_{2}\right) \\
& -\frac{\left(q-q^{-1}\right)}{1-x_{2} / x_{1}} F_{2}^{-}\left(x_{2}\right) F_{1}\left(x_{2}\right)+\frac{1}{1-x_{2} / x_{1}} F_{3,1}\left(x_{2}\right) \\
= & \frac{q-q^{-1} x_{2} / x_{1}}{1-x_{2} / x_{1}}\left(F_{2}^{+}\left(x_{1}\right)-\frac{\left(q-q^{-1}\right) x_{1}}{q-q^{-1} x_{2} / x_{1}} F_{2}^{+}\left(x_{2}\right)\right) F_{1}\left(x_{2}\right) \\
= & \frac{q-q^{-1} x_{2} / x_{1}}{1-x_{2} / x_{1}} F_{2}^{+}\left(x_{1} ; x_{2}\right) F_{1}\left(x_{2}\right)
\end{aligned}
$$

where we have used the definition of the negative half-current (4.12), the expression of the total composed current (3.23) and the Ding-Frenkel relation $F_{2}\left(x_{2}\right)=F_{2}^{+}\left(x_{2}\right)-F_{2}^{-}\left(x_{2}\right)$. 
After substituting formulas (4.17) and (4.18) into integral formulas for the projections of the string (4.15) and (4.16), we obtain, from the resolution of the hierarchical relations for the universal Bethe vectors (4.4) and (4.5), the following intermediate results

$$
\begin{aligned}
P_{e}^{+} & \left(E_{2}\left(\sigma_{b}\right) \cdots E_{2}\left(\sigma_{1}\right) E_{1}\left(\tau_{a}\right) \cdots E_{1}\left(\tau_{1}\right)\right)=\sum_{m=0}^{\min \{a, b\}} \frac{\left(q^{-1}-q\right)^{m}}{m !(a-m) !(b-m) !} \\
& \times \overline{\operatorname{Sym}}_{\bar{\tau}, \bar{\sigma}}\left(\prod_{\substack{1 \leq i<j \leq a-m \\
a-m<i<j \leq a}} \frac{\tau_{i}-\tau_{j}}{q^{-1} \tau_{i}-q \tau_{j}} Y\left(\tau_{a}, \ldots, \tau_{a-m+1} ; \sigma_{m}, \ldots, \sigma_{1}\right)\right. \\
& \times \oint \frac{d \nu_{1}}{\nu_{1}} \cdots \oint \frac{d \nu_{m}}{\nu_{m}} \oint \frac{d \mu_{1}}{\mu_{1}} \cdots \oint \frac{d \mu_{a}}{\mu_{a}} \\
& \times Z\left(\tau_{a}, \ldots, \tau_{1} ; \mu_{a}, \ldots, \mu_{1}\right) Z\left(\mu_{a}, \ldots, \mu_{a-m+1} ; \nu_{1}, \ldots, \nu_{m}\right) \\
& \left.\times P_{e}^{+}\left(E_{2}\left(\sigma_{b}\right) \cdots E_{2}\left(\sigma_{m+1}\right)\right) E_{1}\left(\mu_{1}\right) \cdots E_{1}\left(\mu_{a}\right) E_{2}\left(\nu_{m}\right) \cdots E_{2}\left(\nu_{1}\right)\right)
\end{aligned}
$$

and

$$
\begin{aligned}
P_{f}^{+} & \left(F_{1}\left(t_{1}\right) \cdots F_{1}\left(t_{a}\right) F_{2}\left(s_{1}\right) \cdots F_{2}\left(s_{b}\right)\right)=\sum_{k=0}^{\min \{a, b\}} \frac{\left(q^{-1}-q\right)^{k}}{k !(a-k) !(b-k) !} \\
& \times \overline{\operatorname{Sym}}_{\bar{t}, \bar{s}}\left(\prod_{\substack{1 \leq i<j \leq a-k \\
a-k<i<j \leq a}} \frac{t_{i}-t_{j}}{q^{-1} t_{i}-q t_{j}} Z\left(t_{a}, \ldots, t_{a-k+1} ; s_{k}, \ldots, s_{1}\right)\right. \\
& \times \oint \frac{d y_{1}}{y_{1}} \cdots \oint \frac{d y_{k}}{y_{k}} \oint \frac{d x_{1}}{x_{1}} \cdots \oint \frac{d x_{a}}{x_{a}} \\
& \times Y\left(t_{a}, \ldots, t_{1} ; x_{a}, \ldots, x_{1}\right) Y\left(x_{a}, \ldots, x_{a-k+1} ; y_{1}, \ldots, y_{k}\right) \\
& \left.\times F_{2}\left(y_{1}\right) \cdots F_{2}\left(y_{k}\right) F_{1}\left(x_{a}\right) \cdots F_{1}\left(x_{1}\right) P_{f}^{+}\left(F_{2}\left(s_{k+1}\right) \cdots F_{2}\left(s_{b}\right)\right)\right) .
\end{aligned}
$$

The last step is to move to the left, in (4.20), the product of the total currents $E_{1}\left(\mu_{1}\right) \cdots E_{1}\left(\mu_{a}\right)$ through the projection $P_{e}^{+}\left(E_{2}\left(\sigma_{b}\right) \cdots E_{2}\left(\sigma_{m+1}\right)\right)$ using the factorization formulas (4.11) and the commutation relations (4.19). Analogously, in (4.21), one has to move to the right the product of the total currents $F_{1}\left(x_{a}\right) \cdots F_{1}\left(x_{1}\right)$ through the projection $P_{f}^{+}\left(F_{2}\left(s_{k+1}\right) \cdots F_{2}\left(s_{b}\right)\right)$, using again the factorization formulas (4.11) and the commutation relations (4.19). As result, we obtain the integral formulas (4.8) for the projections of the product of currents for the algebra $U_{q}\left(\widehat{\mathfrak{g l}}_{3}\right)$.

\section{Scalar products of universal Bethe vectors}

\subsection{Commutation of products of total currents}

Formulas (4.8) show that in order to calculate the scalar product of the universal Bethe vectors, one has to commute the products of the total currents

$$
\mathcal{E}(\bar{\mu}, \bar{\nu})=E_{1}\left(\mu_{1}\right) \cdots E_{1}\left(\mu_{a}\right) E_{2}\left(\nu_{b}\right) \cdots E_{2}\left(\nu_{1}\right)
$$

and

$$
\mathcal{F}(\bar{x}, \bar{y})=F_{2}\left(y_{1}\right) \cdots F_{2}\left(y_{b}\right) F_{1}\left(x_{a}\right) \cdots F_{1}\left(x_{1}\right) .
$$


According to the decomposition of the quantum affine algebra $U_{q}\left(\widehat{\mathfrak{g l}}_{3}\right)$ used in this paper, the modes of the total currents $F_{i}[n], E_{i}[n+1], k_{j}^{+}[n], n \geq 0$ and a $q$-commutator $E_{1,3}[1]=$ $E_{2}[0] E_{1}[1]-q^{-1} E_{1}[1] E_{2}[0]$, belong to the Borel subalgebra $U_{q}\left(\mathfrak{b}^{+}\right) \in U_{q}\left(\widehat{\mathfrak{g l}}_{3}\right)$. We define the following ideals in this Borel subalgebra.

Definition 1. We note $J$, the left ideal of $U_{q}\left(\mathfrak{b}^{+}\right)$generated by all elements of the form $U_{q}\left(\mathfrak{b}^{+}\right)$. $E_{i}[n], n>0$ and $U_{q}\left(\mathfrak{b}^{+}\right) \cdot E_{1,3}[1]$. Equalities in $U_{q}\left(\mathfrak{b}^{+}\right)$modulo element from the ideal $J$ are denoted by the symbol ' $\sim_{J}$ '.

Definition 2. Let $I$ be the right ideal of $U_{q}\left(\mathfrak{b}^{+}\right)$generated by all elements of the form $F_{i}[n]$. $U_{q}\left(\mathfrak{b}^{+}\right)$such that $n \geq 0$. We denote equalities modulo elements from the ideal $I$ by the symbol ' $\sim_{I}$ '.

We also define the following ideal in $U_{q}\left(\widehat{\mathfrak{g l}}_{3}\right)$ :

Definition 3. We denote by $K$ the two-sided $U_{q}\left(\widehat{\mathfrak{g l}}_{3}\right)$ ideal generated by the elements which have at least one arbitrary mode $k_{j}^{-}[n], n \leq 0$, of the negative Cartan current $k_{j}^{-}(t)$. Equalities in $U_{q}\left(\widehat{\mathfrak{g l}}_{3}\right)$ modulo element of the ideal $K$ are denoted by the symbol ' $\sim_{K}$ '.

Equalities in $U_{q}\left(\widehat{\mathfrak{g l}}_{3}\right)$ modulo the right ideal $I$, the left ideal $J$ and the two-sided ideal $K$ will be denoted by the symbol ' $\approx$ '.

A right weight singular vector defined by the relations (2.7) is annihilated by the right action of any positive mode $E_{i}[n], n>0$, the element $E_{1,3}[1]$ and is a right-eigenvector for $k_{j}^{+}(t)$,

$$
E_{i}^{+}(\tau) \cdot v=0, \quad P_{e}^{+}\left(E_{1,3}(\tau)\right) \cdot v=0, \quad k_{j}^{+}(\tau) \cdot v=\Lambda_{j}(\tau) v
$$

where $\Lambda_{j}(\tau)$ are some meromorphic functions, decomposed as a power series in $\tau^{-1}$. A left weight singular vector $v^{\prime}$ defined by the relation (2.9) is annihilated by the left action of any nonnegative modes $F_{i}[n], n \geq 0$ and is a left-eigenvector for $k_{j}^{+}(t)$,

$$
v^{\prime} \cdot F_{i}^{+}(t)=0, \quad v^{\prime} \cdot k_{j}^{+}(t)=\Lambda_{j}^{\prime}(t) v^{\prime}
$$

where $\Lambda_{j}^{\prime}(t)$ are also meromorphic functions. These facts follow from the relation between projections of the currents and the Gauss coordinates of the L-operator (3.1)-(3.3).

We observe that the vectors

$$
P_{f}^{+}\left(F_{1}\left(t_{1}\right) \cdots F_{1}\left(t_{a}\right) F_{2}\left(s_{1}\right) \cdots F_{2}\left(s_{b}\right)\right) \cdot v
$$

and

$$
v^{\prime} \cdot P_{e}^{+}\left(E_{2}\left(\sigma_{b}\right) \cdots E_{2}\left(\sigma_{1}\right) E_{1}\left(\tau_{a}\right) \cdots E_{1}\left(\tau_{1}\right)\right)
$$

belong to the modules over the quantum affine algebra $U_{q}\left(\widehat{\mathfrak{g l}}_{3}\right)$ from the categories of the highest weight and lowest weight representations respectively. This is in accordance with the definition of the completions $\bar{U}_{E}$ and $\bar{U}_{F}$ and the corresponding projections given above.

We assume the existence of a nondegenerate pairing $\left\langle v^{\prime}, v\right\rangle$ and by the scalar product of the left and right universal Bethe vectors, we will understand the coefficient $\mathcal{S}(\bar{\tau}, \bar{\sigma} ; \bar{t}, \bar{s})$ in front of the pairing $\left\langle v^{\prime}, v\right\rangle$ in the right hand side of equality

$$
\begin{aligned}
& \left\langle v^{\prime} \cdot P_{e}^{+}\left(E_{2}\left(\sigma_{b}\right) \cdots E_{2}\left(\sigma_{1}\right) E_{1}\left(\tau_{a}\right) \cdots E_{1}\left(\tau_{1}\right)\right), P_{f}^{+}\left(F_{1}\left(t_{1}\right) \cdots F_{1}\left(t_{a}\right) F_{2}\left(s_{1}\right) \cdots F_{2}\left(s_{b}\right)\right) \cdot v\right\rangle \\
& \quad=\mathcal{S}\left(\tau_{1}, \ldots, \tau_{a}, \sigma_{1}, \ldots, \sigma_{b} ; t_{1}, \ldots, t_{a}, s_{1}, \ldots, s_{b}\right)\left\langle v^{\prime}, v\right\rangle .
\end{aligned}
$$


It is clear that the scalar product (2.11) differs from (5.3) by the product

$$
\prod_{k=1}^{a} \Lambda_{1}\left(t_{k}\right) \Lambda_{1}^{\prime}\left(\tau_{k}\right) \prod_{m=1}^{b} \Lambda_{2}\left(s_{m}\right) \Lambda_{2}^{\prime}\left(\sigma_{m}\right)
$$

The problem of calculation of the scalar product of the universal Bethe vectors (5.3) is equivalent to the commutation of the projections entering the definitions of the vectors (5.1) and (5.2) modulo the left ideal $J$ and the right ideal $I$. To calculate this commutation, we use the integral presentation of the projections (4.8), commute the total currents and then calculate the integrals. Since both projections belong to the positive Borel subalgebra $U_{q}\left(\mathfrak{b}^{+}\right)$, we can neglect the terms which contain the negative Cartan currents $k_{i}^{-}(t)$ and perform the commutation of the total currents modulo the two-sided ideal $K$. Actually, in commuting the total currents, we will be interested only in terms which are products of combinations of the $U_{q}\left(\widehat{\mathfrak{g l}}_{3}\right)$ positive Cartan currents (3.17). All other terms will be annihilated by the weight singular vectors.

Let us recall that elements $\mathcal{E}(\bar{\mu}, \bar{\nu})$ and $\mathcal{F}(\bar{x}, \bar{y})$ are elements of the completed algebras $\bar{U}_{E}$ and $\bar{U}_{F}$, which are dual subalgebras in $U_{q}\left(\widehat{\mathfrak{g l}}_{3}\right)$ considered as a quantum double. There is a nondegenerate Hopf pairing between these subalgebras, given by the formulas (3.16). For any elements $a \in \mathcal{A}$ and $b \in \mathcal{B}$ from two dual Hopf subalgebras $\mathcal{A}$ and $\mathcal{B}$ of the quantum double algebra $\mathcal{D}(\mathcal{A})=\mathcal{A} \oplus \mathcal{B}$, there is a relation $[26]$

$$
\left\langle a^{(2)}, b^{(2)}\right\rangle b^{(1)} \cdot a^{(1)}=a^{(2)} \cdot b^{(2)}\left\langle a^{(1)}, b^{(1)}\right\rangle,
$$

where $\Delta_{\mathcal{A}}(a)=a^{(1)} \otimes a^{(2)}$ and $\Delta_{\mathcal{B}}(b)=b^{(1)} \otimes b^{(2)}$.

Let us apply formula $(5.4)$ for $a=\mathcal{E}(\bar{\mu}, \bar{\nu})=\mathcal{E}$ and $b=\mathcal{F}(\bar{x}, \bar{y})=\mathcal{F}$. Using the current coproduct (3.15), we conclude that

$$
\Delta^{(D)} \mathcal{E}=1 \otimes \mathcal{E}+\mathcal{E}^{\prime} \otimes \mathcal{E}^{\prime \prime}, \quad \Delta^{(D)} \mathcal{F}=\mathcal{K}^{+} \otimes \mathcal{F}+\mathcal{F}^{\prime} \otimes \mathcal{F}^{\prime \prime},
$$

where the element $\mathcal{E}^{\prime}$ satisfies $\varepsilon\left(\mathcal{E}^{\prime}\right)=0$ and the element $\mathcal{E}^{\prime \prime}$ contains at least one negative Cartan current $k_{i}^{-}(\tau)$. The element $\mathcal{K}^{+}$in (5.5) takes the form

$$
\mathcal{K}^{+}=\prod_{i=1}^{a} \psi_{1}^{+}\left(x_{i}\right) \prod_{j=1}^{b} \psi_{2}^{+}\left(y_{j}\right) .
$$

The left hand side of the relation (5.4) have the form

$$
\langle\mathcal{E}, \mathcal{F}\rangle \cdot \mathcal{K}^{+} \bmod \tilde{J}
$$

and the right hand side of the same relation is

$$
\mathcal{E} \cdot \mathcal{F} \bmod K
$$

The ideal $\tilde{J}$, similar to the ideal $J$, is the left ideal in $U_{q}\left(\widehat{\mathfrak{g l}}_{3}\right)$ generated by the elements $U_{q}\left(\widehat{\mathfrak{g l}}_{3}\right)$. $E_{i}[n], i=1,2$ and $n \in \mathbb{Z}$. One can check that after integration in (4.8) the terms of the ideal $\tilde{J}$ which have non-positive modes of the currents $E_{1}\left(\mu_{k}\right)$ and $E_{2}\left(\nu_{m}\right)$ on the right will disappear and can be neglected. Alternatively, we can argue that these terms are irrelevant using cyclic ordering of the current or Cartan-Weyl generators, as it was done in the papers [26, 21].

As result, a general equality (5.4) for the given elements $a=\mathcal{E}(\bar{\mu}, \bar{\nu})$ and $b=\mathcal{F}(\bar{x}, \bar{y})$ reads

$$
\mathcal{E}(\bar{\mu}, \bar{\nu}) \cdot \mathcal{F}(\bar{x}, \bar{y})=\langle\mathcal{E}(\bar{\mu}, \bar{\nu}), \mathcal{F}(\bar{x}, \bar{y})\rangle \prod_{i=1}^{a} \psi_{1}^{+}\left(x_{i}\right) \prod_{j=1}^{b} \psi_{2}^{+}\left(y_{j}\right) \quad \bmod (K, J)
$$

modulo ideals $K$ and $J$. This relation shows that instead of calculating the exchange relations for the product of the currents $\mathcal{E}(\bar{\mu}, \bar{\nu})$ and $\mathcal{F}(\bar{x}, \bar{y})$ it is enough to calculate the pairing between them. 


\subsection{Pairing and integral formula for scalar products}

To calculate the pairing, we will use the basic properties of pairing between dual Hopf subalgebras

$$
\left\langle a_{1} a_{2}, b\right\rangle=\left\langle a_{1} \otimes a_{2}, \Delta_{\mathcal{B}}(b)\right\rangle, \quad\left\langle a, b_{1} b_{2}\right\rangle=\left\langle\Delta_{\mathcal{A}}(a), b_{2} \otimes b_{1}\right\rangle,
$$

where $\mathcal{A}=U_{E}$ and $\mathcal{B}=U_{F}$. From these properties, we obtain

$$
\begin{aligned}
\langle\mathcal{E}(\bar{\mu}, \bar{\nu}), \mathcal{F}(\bar{x}, \bar{y})\rangle= & \prod_{i=1}^{b} \prod_{j=1}^{a} \frac{q^{-1} x_{j}-q y_{i}}{x_{j}-y_{i}} \prod_{i<j}^{a} \frac{q^{-1} x_{i}-q x_{j}}{q x_{i}-q^{-1} x_{j}} \prod_{i<j}^{b} \frac{q^{-1} y_{i}-q y_{j}}{q y_{i}-q^{-1} y_{j}} \\
& \times\left(q-q^{-1}\right)^{a+b} \overline{\operatorname{Sym}}_{\bar{x}}\left(\prod_{i=1}^{a} \delta\left(\mu_{i} / x_{i}\right)\right) \overline{\operatorname{Sym}}_{\bar{y}}\left(\prod_{i=1}^{b} \delta\left(\nu_{i} / y_{i}\right)\right) .
\end{aligned}
$$

Using the definition of the scalar product of the universal Bethe vectors (5.3) and integral presentations of the projections (4.8), we conclude

\section{Proposition 3.}

$$
\begin{aligned}
\mathcal{S}\left(\tau_{1}, \ldots, \tau_{a}, \sigma_{1}, \ldots, \sigma_{b} ; t_{1}, \ldots, t_{a}, s_{1}, \ldots, s_{b}\right) \\
=\left(q-q^{-1}\right)^{a+b} \oint \frac{d x_{1}}{x_{1}} \ldots \oint \frac{d x_{a}}{x_{a}} \oint \frac{d y_{1}}{y_{1}} \cdots \oint \frac{d y_{b}}{y_{b}} \prod_{i=1}^{b} \prod_{j=1}^{a} \frac{q^{-1} x_{j}-q y_{i}}{x_{j}-y_{i}} \\
\times \prod_{i<j}^{a} \frac{q^{-1} x_{i}-q x_{j}}{q x_{i}-q^{-1} x_{j}} \prod_{i<j}^{b} \frac{q^{-1} y_{i}-q y_{j}}{q y_{i}-q^{-1} y_{j}} \mathbb{E}(\bar{\tau}, \bar{\sigma} ; \bar{x}, \bar{y}) \overline{\operatorname{Sym}}_{\bar{x}, \bar{y}}(\mathbb{F}(\bar{t}, \bar{s} ; \bar{x}, \bar{y})) \\
\times \prod_{i=1}^{a} \psi_{1}^{+}\left(x_{i}\right) \prod_{j=1}^{b} \psi_{2}^{+}\left(y_{j}\right),
\end{aligned}
$$

where the rational series $\mathbb{E}(\bar{\tau}, \bar{\sigma} ; \bar{x}, \bar{y})$ and $\mathbb{F}(\bar{t}, \bar{s} ; \bar{x}, \bar{y})$ are given in (4.9) and (4.10).

\section{Conclusions}

The kernels entering the formulas (4.8) can be $q$-symmetrized over integration variables due to the $q$-symmetric properties of the product of the total currents. In the $\mathfrak{g l}_{2}$ case, this leads to the determinant representation of the kernel due to the identity

$$
\begin{gathered}
\prod_{i<j}^{n} \frac{q^{-1} t_{i}-q t_{j}}{t_{i}-t_{j}} \overline{\operatorname{Sym}}_{\bar{t}}(Y(\bar{t}, \bar{x}))=\prod_{i<j}^{n} \frac{q^{-1} x_{i}-q x_{j}}{x_{i}-x_{j}} \overline{\operatorname{Sym}}_{\bar{x}}(Y(\bar{t}, \bar{x})) \\
=\frac{\prod_{i} t_{i} \prod_{i, j}\left(q^{-1} t_{i}-q x_{j}\right)}{\prod_{i<j}\left(t_{i}-t_{j}\right)\left(x_{j}-x_{i}\right)} \operatorname{det}\left|\frac{1}{\left(t_{i}-x_{j}\right)\left(q^{-1} t_{i}-q x_{j}\right)}\right|_{i, j=1, \ldots, n},
\end{gathered}
$$

where the determinant on the right hand side is called an Izergin determinant. It is equal (up to a scalar factor) to the partition function of the $X X Z$ model with domain wall boundary conditions [8].

The challenge is to get determinant formulas for the $q$-symmetrized kernels (4.9) and (4.10) as a sum of determinants and to use further this determinant formula to get a determinant formula for the scalar products. Work in this direction is in progress. 


\section{Acknowledgement}

Authors would like to acknowledge very useful discussions with Sergei Khoroshkin and Nikita Slavnov. This work was partially done when the second author (S.P.) visited Laboratoire d'Annecy-Le-Vieux de Physique Théorique in February, 2009. This visit was possible due to the financial support of the CNRS-Russia exchange program on mathematical physics. He thanks LAPTH for the hospitality and stimulating scientific atmosphere. Work of S.P. was supported in part by RFBR grant 08-01-00667, RFBR-CNRS grant 07-02-92166-CNRS and grant of the Federal Agency for Science and Innovations of Russian Federation under contract 14.740.11.0347. Work of S.B. was supported in part by the INFN Iniziativa Specifica FI11.

\section{References}

[1] Bethe H., Zur Theorie der Metalle. I. Eigenwerte und Eigenfunktionen der linearen Atomkette, Z. Phys. 71 (1931), 205-226.

[2] Takhtajan L.A., Faddeev L.D., The quantum method of the inverse problem and the Heisenberg $X Y Z$ model, Russian Math. Surveys 34 (1979), no. 5, 11-68.

[3] Sklyanin E.K., Faddeev L.D., Quantum mechanical approach to completely integrable field theory models, Sov. Phys. Dokl. 23 (1978), 902-904.

[4] Sklyanin E.K., Takhtajan L.A., Faddeev L.D., Quantum inverse problem method. I, Theoret. and Math. Phys. 40 (1980), 688-706.

[5] Faddeev L.D., How algebraic Bethe ansatz works for integrable models, in Symétries Quantiques (Les Houches, 1995), Editors A. Connes et al., North-Holland, Amsterdam, 1998, 149-219, hep-th/9605187.

[6] Korepin V.E., Calculation of norms of Bethe wave functions, Comm. Math. Phys. 86 (1982), 391-418.

[7] Izergin A.G., Korepin V.E., The quantum inverse scattering method approach to correlation functions, Comm. Math. Phys. 94 (1984), 67-92.

[8] Izergin A.G., Partition function of a six-vertex model in a finite volume, Soviet Phys. Dokl. 32 (1987), 878-879.

[9] Slavnov N.A., Calculation of scalar products of wave functions and form factors in the framework of the algebraic Bethe ansatz, Theoret. and Math. Phys. 79 (1989), 502-508.

[10] Kojima T., Korepin V.E., Slavnov N.A., Determinant representation for dynamical correlation functions of the quantum nonlinear Schrödinger equation, Comm. Math. Phys. 188 (1997), 657-689, hep-th/9611216.

[11] Izergin A.G., Kitanine N., Maillet J.M., Terras V., Spontaneous magnetization of the $X X Z$ Heisenberg spin-1/2 chain, Nuclear Phys. B 554 (1999), 679-696, solv-int/9812021.

[12] Kitanine N., Maillet J.M., Terras V., Correlation functions of the $X X Z$ Heisenberg spin- $1 / 2$ chain in a magnetic field, Nuclear Phys. B 567 (2000), 554-582, math-ph/9907019.

[13] Kitanine N., Maillet J.M., Slavnov N.A., Terras V., Master equation for spin-spin correlation functions of the $X X Z$ chain, Nuclear Phys. B 712 (2005), 600-622, hep-th/0406190.

[14] Kulish P.P., Reshetikhin N.Yu., Diagonalization of GL( $N)$ invariant transfer matrices and quantum $N$-wave system (Lee model), J. Phys. A: Math. Gen. 16 (1983), L591-L596.

[15] Belliard S., Ragoucy E., The nested Bethe ansatz for "all" closed spin chains, J. Phys. A: Math. Theor. 41 (2008), 295202, 33 pages, arXiv:0804.2822.

[16] Varchenko A.N., Tarasov V.O., Jackson integrals for the solutions to Knizhnik-Zamolodchikov equation, St. Petersburg Math. J. 2 (1995), 275-313.

[17] Tarasov V., Varchenko A., Combinatorial formulae for nested Bethe vectors, math.QA/0702277.

[18] Drinfel'd V.G., New realization of Yangians and of quantum affine algebras, Sov. Math. Dokl. 36 (1988), 212-216.

[19] Ding J.T., Frenkel I.B., Isomorphism of two realizations of quantum affine algebra $U_{q}\left(\widehat{\mathfrak{g l}}_{N}\right)$, Comm. Math. Phys. 156 (1993), 277-300.

[20] Khoroshkin S., Pakuliak S., A computation of an universal weight function for the quantum affine algebra $U_{q}\left(\widehat{\mathfrak{g l}}_{N}\right)$, J. Math. Kyoto Univ. 48 (2008), 277-321, arXiv:0711.2819. 
[21] Frappat L., Khoroshkin S., Pakuliak S., Ragoucy E., Bethe ansatz for the universal weight function, Ann. Henri Poincaré 10 (2009), 513-548, arXiv:0810.3135.

[22] Arnaudon D., Crampé N., Doikou A., Frappat, L., Ragoucy E. Spectrum and Bethe ansatz equations for the $U_{q}(g l(\mathcal{N}))$ closed and open spin chains in any representation, Ann. Henri Poincaré 7 (2006), 1217-1268, math-ph/0512037.

[23] Slavnov N.A., The algebraic Bethe ansatz and quantum integrable systems, Russian Math. Surveys 62 (2007), 727-766.

[24] Os'kin A., Pakuliak S., Silantyev A., On the universal weight function for the quantum affine algebra $U_{q}\left(\widehat{\mathfrak{g l}}_{N}\right)$, St. Petersburg Math. J. 21 (2010), 651-680, arXiv:0711.2821.

[25] Enriquez B., Rubtsov V., Quasi-Hopf algebras associated with $\mathfrak{s l}_{2}$ and complex curves, Israel J. Math. 112 (1999), 61-108, q-alg/9608005.

[26] Enriquez B., Khoroshkin S., Pakuliak S., Weight functions and Drinfeld currents, Comm. Math. Phys. 276 (2007), 691-725, math.QA/0610398.

[27] Khoroshkin S., Pakuliak S., Tarasov V., Off-shell Bethe vectors and Drinfeld currents, J. Geom. Phys. 57 (2007), 1713-1732, math.QA/0610517.

[28] Pakuliak S.Z., Khoroshkin S.M., The weight function for the quantum affine algebra $U_{q}\left(\widehat{\mathfrak{s l}}_{3}\right)$, Theoret. and Math. Phys. 145 (2005), 1373-1399, math.QA/0610433. 